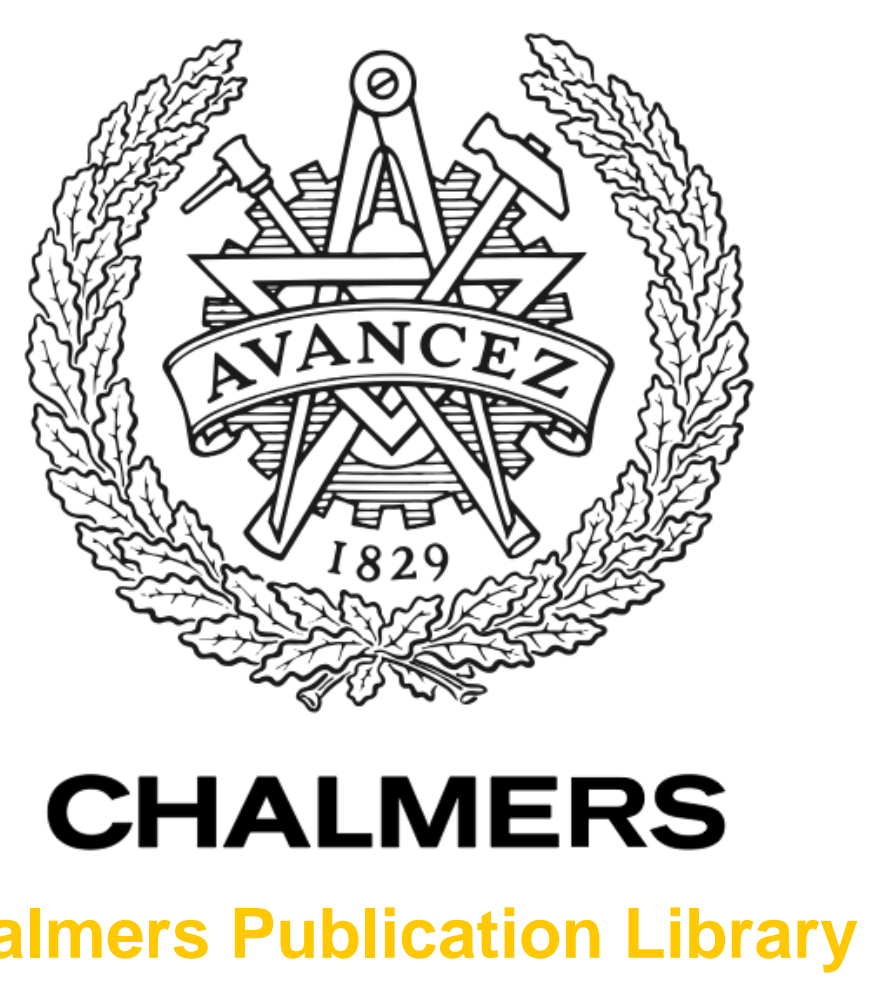

Chalmers Publication Library

\title{
Design and Validation of Microstrip Gap Waveguides and Their Transitions to Rectangu-lar Waveguide, for Millimeter-Wave Applications
}

This document has been downloaded from Chalmers Publication Library (CPL). It is the author's version of a work that was accepted for publication in:

IEEE Transactions on Microwave Theory and Techniques (ISSN: 0018-9480)

Citation for the published paper:

Algaba Brazález, A. ; Rajo-Iglesias, E. ; Vázquez-Roy, J. et al. (2015) "Design and Validation of Microstrip Gap Waveguides and Their Transitions to Rectangu-lar Waveguide, for Millimeter-Wave Applications". IEEE Transactions on Microwave Theory and Techniques, vol. 63(12), pp. 4035-4050.

http://dx.doi.org/10.1109/TMTT.2015.2495141

Downloaded from: http://publications.lib.chalmers.se/publication/227713

Notice: Changes introduced as a result of publishing processes such as copy-editing and formatting may not be reflected in this document. For a definitive version of this work, please refer to the published source. Please note that access to the published version might require a subscription. 


\title{
Design and Validation of Microstrip Gap Waveguides and Their Transitions to Rectangular Waveguide, for Millimeter-Wave Applications
}

\author{
Astrid Algaba Brazález, Eva Rajo-Iglesias, Senior Member, IEEE, Jose-Luis Vazquez-Roy, Member, IEEE, Abbas \\ Vosoogh, and Per-Simon Kildal, Fellow, IEEE
}

\begin{abstract}
The paper describes the design methodology, experimental validation and practical considerations of two millimeterwave wideband vertical transitions from two gap waveguide versions (inverted microstrip gap waveguide, and microstrip packaged by using gap waveguide) to standard WR-15 rectangular waveguide. The experimental results show $S_{11}$ smaller than $-10 \mathrm{~dB}$ over relative bandwidths larger than $25 \%$ and $26.6 \%$ when Rogers $\mathrm{RO3003}$ and $\mathrm{RO4003}$ materials are used respectively. The vertical transition from standard microstrip line packaged by a lid of pins to WR-15, shows measured return loss better than $15 \mathrm{~dB}$ over $13.8 \%$ relative bandwidth. The new transitions can be used as interfaces between gap waveguide feed-networks for $60 \mathrm{GHz}$ antenna systems, testing equipment (like Vector Network Analyzers) and components with WR-15 ports, such as transmitting/receiving amplifiers. Moreover, the paper documents the losses of different gap waveguide prototypes compared to unpackaged microstrip line and Substrate Integrated Waveguide (SIW). This investigation shows that in V-band the lowest losses are achieved with inverted microstrip gap waveguide.
\end{abstract}

Index Terms-Artificial Magnetic Conductor (AMC), dissipation loss, feed network, gap waveguide, microstrip, millimeterwaves, packaging, Perfect Magnetic Conductor (PMC), rectangular waveguide, transition.

\section{INTRODUCTION}

$\mathbf{T}$ HERE is a growing amount of applications at the millimeter-wave frequency band $(30-300 \mathrm{GHz})$, such as high data rate wireless communications (operating over the unlicensed frequency band between 57 and $64 \mathrm{GHz}$ ), and automotive radar systems (which operate from 76 to $81 \mathrm{GHz}$ ). This has motivated the need for developing new technologies suitable to cost-effectively fulfill the stricter tolerance requirements at these frequencies.

Common planar circuit technologies such as microstrip or coplanar waveguide (CPW) are typically used at millimeterwave frequencies for integration of active and passive components, and for designing millimeter-wave planar array antennas. Low cost, light weight, compact profile and easy manufacturing are the key factors that make planar structures attractive

A. A. Brazález, Abbas Vosoogh and P.-S. Kildal are with the Department of Signals and Systems at Chalmers University of Technology, Göteborg, Sweden. (e-mail: astrid.algaba@chalmers.se, per-simon.kildal@chalmers.se).

E. Rajo-Iglesias and J.-L. Vázquez-Roy are with the Department of Signal Theory and Communication, University Carlos III of Madrid, Madrid, Spain

Manuscript submitted March 10, 2015. This work was supported by the next 4 parties: the Swedish Research Council VR, by the Swedish Governmental Agency for Innovation Systems VINNOVA via a project within the VINN Excellence center Chase, the European Research Council (ERC) via the advanced investigator grant ERC-2012-ADG-20120216, and the Spanish Government under project TEC2013-44019-R. for high frequency applications. However, they experience high conductive and dielectric losses, radiation leakage and presence of surface waves [1]. The losses become especially critical when designing microstrip feed networks [2]. The consequences of all these limitations are a considerable reduction in the gain and antenna efficiency, as well as high side-lobes.

On the other hand, hollow waveguide slot arrays show low loss, high gain and high radiation efficiency. Still, the manufacturing of multilayer waveguide distribution feed networks [3]- [4], and the integration of active and passive components becomes very challenging as the operating frequency increases. The reason is the severe tolerances requirements to ensure good conducting joints between the split metal blocks composing the waveguide.

Substrate Integrated Waveguide (SIW) constitutes a planar printed circuit board (PCB) solution by which the substrate has ground planes on both sides, and waveguides are formed between rows of metalized via holes embedded in the substrate [5]- [6]. SIW has electromagnetic characteristics similar to standard hollow waveguides, and at the same time preserving the advantages of PCB technology. Lot of research has been done during the past ten years on the design of passive components and antennas in SIW technology [7]- [9]. However, SIWs suffer from the disadvantage of higher loss than hollow waveguides due to the unavoidable loss tangent of the dielectric. Low loss substrates can become expensive and they are often mechanically soft, which will increase the cost of providing via holes when mass-produced. Moreover, the design of SIW components may become a complex process due to the need of fine-tuning of the placement of the large amount of via holes present in the SIW circuits.

A new guiding structure called gap waveguide was proposed to overcome the above-mentioned limitations of the traditional technologies used at millimeter-wave frequencies (microstrip, standard waveguides and SIW). The theoretical background of the gap waveguide technology can be found in [10]- [13], and the verification by measurements of a first ridge gap waveguide demonstrator operating in $\mathrm{Ku}$-band was presented in [14]. The gap waveguide approach is able to merge the benefits of the traditional millimeter-wave technologies. Firstly, it has low losses [15] because dielectric material is not really needed. In addition, the fields are strictly confined within the air gap, thereby allowing for an increase of the line dimensions resulting in a reduction of the conductive losses. Another relevant factor is that the gap structure is properly packaged 
by itself, since the employed periodic structure together with the smooth metal plate removes any possible leakage. Furthermore, the non-conductive assembly of the two metal plates of gap prototypes makes them more flexible and cost-effective to manufacture than hollow waveguides.

The integration of active RF parts, passive components and antennas in the same gap waveguide millimeter-wave module is our overall goal. This integration requires compatibility towards both planar structures (microstrip, CPW) and rectangular waveguides. The reason is that Monolithic Microwave Integrated Circuits (MMIC) are substrate-based and their inputs/outputs are typically $50 \Omega$ planar transmission lines. Some good transitions to microstrip/CPW have been already studied in order to integrate MMIC chips into gap waveguides [16]-[18]. On the other hand, millimeter-wave measurement equipment is usually standardized to rectangular waveguides and CPW, the latter for Ground-Signal-Ground probe stations. In [17] a vialess air-bridge transition from CPW to ridge gap waveguide operating at $100 \mathrm{GHz}$ was proposed, showing a return loss smaller than $15 \mathrm{~dB}$ over almost $10 \%$ bandwidth.

In the present paper we introduce two wideband and compact gap waveguide transitions to standard WR-15 rectangular waveguide operating in the $60 \mathrm{GHz}$ band. Sections II-VI deal with a first transition design that interconnects an inverted microstrip gap waveguide to WR-15. Sections VII-VIII introduce the geometry of a transition from microstrip packaged by a lid of nails, to a WR-15. Section IX presents a millimeter-wave comparative study of loss of the two gap waveguide versions mentioned before. We also include two additional cases in the comparison: an unpackaged microstrip line and a substrate integrated waveguide, both operating in the $60 \mathrm{GHz}$ band.

\section{INVERTED MICROSTRIP GAP WAVEGUIDE}

The inverted microstrip gap waveguide technology is based on the presence of a thin substrate that lies over a periodic pin pattern, i.e., a bed of nails [19]- [20]. This bed of nails constitutes an Artificial Magnetic Conductor (AMC) material, which combined with a smooth metal plate defines a cutoff of all parallel-plate modes and surface waves within an air gap (distance between the bed of nails and the metal plate, that should be smaller than quarter wavelength). Thereby, all waves are prohibited also in the presence of the dielectric layer. Only local waves are allowed to propagate along strips etched on this substrate. The suppression of parallel-plate modes and surface waves is ensured within a certain frequency band referred to as a stopband [21]. Fig. 1 shows the basic layout of the inverted microstrip gap waveguide.

Since our transition needs to cover the whole unlicensed $60 \mathrm{GHz}$ frequency band, the pin dimensions of the inverted microstrip gap waveguide have been suitably chosen to reach a stopband that includes as much as possible of the $\mathrm{V}$ band. These dimensions are described in Table I, and Fig. 2(a) shows the corresponding dispersion diagram for the infinite unit cell without metal strip on the Printed Circuit Board (PCB), whereas Fig. 2(b) illustrates the resulting dispersion diagram for the infinite unit cell considering a metal strip on the top side of the PCB. The dispersion diagram has been obtained

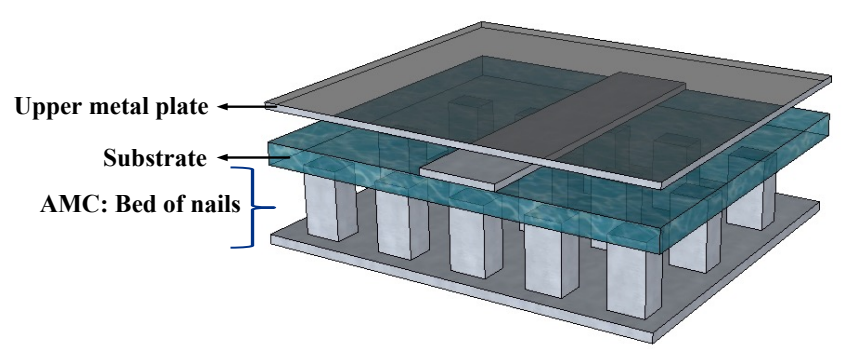

Fig. 1. Basic geometry of the inverted microstrip gap waveguide.

by using the Eigenmode solver of CST Microwave Studio. The considered substrate material is Rogers RO3003 with permittivity $\epsilon_{r}=3$, loss tangent $\tan \delta=0.0013$ (specifications are at $10 \mathrm{GHz}$ according to Rogers material data sheet) and thickness $h=0.25 \mathrm{~mm}$. Fig. 2(a) shows that there is a parallelplate stopband between $50.55 \mathrm{GHz}$ and $75 \mathrm{GHz}$, and Fig. 2(b) illustrates that there is a single propagating mode between 52.4 and $68 \mathrm{GHz}$, which involves the whole $60 \mathrm{GHz}$ frequency band. This is the desired Quasi-TEM mode following the strip. The stopband of the parallel-plate modes appears between 50.55 and $75 \mathrm{GHz}$. The mode appearing at $68 \mathrm{GHz}$ is discussed in more detail in Section IV.

The inverted microstrip gap waveguide technology constitutes an attractive alternative to standard microstrip, especially for designing corporate feed-networks of horn/slot antenna array systems [22]. A critical obstacle so far has been the absence of good transitions that allow connection of the inverted microstrip gap prototypes to measurement equipment at millimeter-wave frequencies. A previous transition investigation [23] shows a wideband behavior, but the rectangular waveguide opening extents vertically upwards from the top side of the PCB. The best option for antenna applications would be to use vertical transitions that extent downwards. This means that the rectangular waveguide opening is placed on the bottom side of the PCB, in a similar way as the transition from microstrip ridge gap waveguide to WR-15 presented in [24] used for the gap waveguide feed-network in [25]. In this way, the rectangular waveguide opening is located on the opposite plate compared to that of the radiating elements of the array, and we can allow connection to the transmitting or receiving amplifiers from the back-side of the PCB without affecting the antenna radiation pattern. Reference [26] presents a preliminary downwards transition from inverted microstrip gap waveguide to WR-8, but the performance shows a limited bandwidth (return loss is better than $15 \mathrm{~dB}$ over $10.5 \%$ ). In the next section we will present the design of a new transition that ensures much better compatibility between inverted microstrip gap waveguide and WR-15.

\section{InVERTED Microstrip Gap WAVEguide To WR-15 TRANSITION DESIGN}

We can distinguish between three main types of transitions:

1) Inline transitions (positioned along propagation direction of the waveguide) [27]- [28].

2) Vertical transitions (placed transversally to propagation direction of the waveguide) [29]-[32]. 
TABLE I

DIMENSIONS OF GAP WAVEGUIDE LAYER (REFERRED TO UNIT CELL SHOWN IN FIG.2)

\begin{tabular}{|c|c|}
\hline Parameter & Value \\
\hline Air gap, $\mathrm{g}$ & $0.25 \mathrm{~mm}$ \\
\hline $\mathrm{p}$ & $0.73 \mathrm{~mm}$ \\
\hline $\mathrm{p} 2$ & $0.68 \mathrm{~mm}$ \\
\hline $\mathrm{a}$ & $0.38 \mathrm{~mm}$ \\
\hline $\mathrm{a} 2$ & $0.33 \mathrm{~mm}$ \\
\hline $\mathrm{W}(50 \Omega)$ & $1.11 \mathrm{~mm}$ \\
\hline Height of the pin, $\mathrm{d}$ & $1.05 \mathrm{~mm}$ \\
\hline Thickness of substrate, $\mathrm{h}$ & $0.25 \mathrm{~mm}$ \\
\hline
\end{tabular}

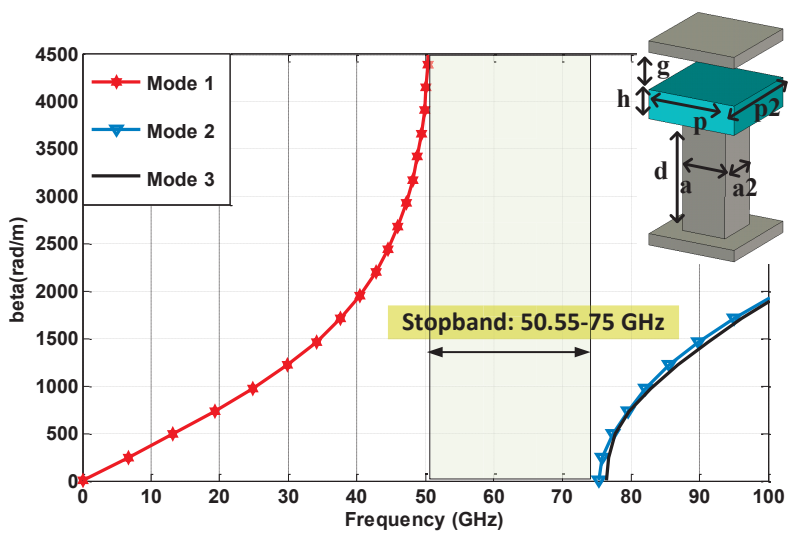

(a)

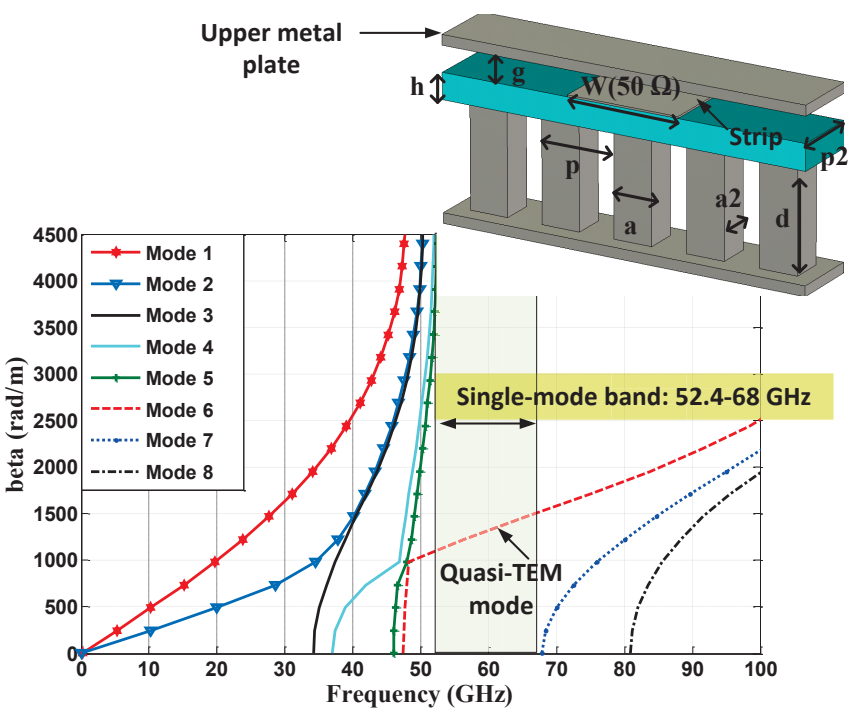

(b)

Fig. 2. (a) Dispersion diagram for the corresponding infinite periodic unit cell without metal strip. (b) Dispersion diagram for the corresponding infinite periodic unit cell including metal strip on the top of the PCB.

3) Aperture coupled patch transitions: the field/impedance matching is achieved via a resonant patch through an aperture in the ground plane of the transmission line. This usually constitutes a subtype within the vertical transitions group [33]- [34].
In [33] and [34] vertical transitions from standard microstrip to rectangular waveguide based on type 3 were described. The main issue of this type of transitions is the potential leakage of parallel-plate modes into the substrate due to the presence of different parallel metal layers. The only way to avoid these modes is by adding via holes which means an increase in the design complexity and manufacturing cost. These metalized via holes are also needed to create the extension of the waveguide walls into the PCB.

In this section we present a wideband vertical transition between inverted microstrip gap waveguide and WR-15. The principle of operation of the here proposed gap waveguide transition is based on well-known E-plane probe transitions that have been widely employed to interconnect microstrip lines with rectangular waveguides [29]-[32]. In our design there is no need to add any via holes to avoid parallel-plate or higher order modes. The reason for this is that any possible excitation of these modes is eliminated by the gap waveguide itself. Moreover, the transition is easily integrated into the rectangular waveguide and no complex modifications or holes in the walls of the waveguide are needed in order to ensure such integration. The transition presented in [29] shows a small hole in the broad wall of the waveguide that allows the insertion of a microstrip probe.

The transition is composed of two main parts. Firstly, a PCB is positioned over a bed of pins and it contains a $50 \Omega$ feeding line terminated by a two-steps tapered matching circuit. These tapered-line sections act as an impedance transformer and are properly placed over the rectangular waveguide opening. A parametric sweep of the position and dimensions of the matching circuit has been carried out in order to achieve optimum return loss within the frequency band under interest. The layout of the transition circuit is shown on Fig. 3 and all parameter values are specified on Table II. On the other hand, the transition geometry is complemented by adding a cavity backshort on the upper metal lid (see cross-section of the complete transition geometry illustrated in Fig. 4). Theoretically, this cavity should be placed at a distance equal to $\lambda / 4$ from the inverted microstrip probe in order to establish an open boundary condition on the PCB plane. In this way, we force the Quasi-TEM fields of the inverted microstrip gap waveguide to propagate down into the waveguide and match the fundamental $T E_{10}$ mode of WR-15. The backshort is positioned opposite to the rectangular waveguide opening and its transversal dimensions are the same as those of the standard WR-15. The distance between the backshort and the substrate was initially set as $\lambda / 4$ and then tuned in order to compensate the reactance introduced by the two-steps matching probe and optimize the transition behavior. Hereby, the backshort together with the tapered line contained in the PCB contributes to provide field matching as well as impedance matching over a wide bandwidth.

\section{Simulated Results And Losses In INVERTED MicrostriP GAP WAVEGUIDE}

This section presents simulation results of three prototypes. Firstly, a transition between inverted microstrip gap waveguide and WR-15 in single configuration (see Fig. 5) is 
TABLE II

DIMENSIONS OF PCB LAYER (REFERRED TO LAYOUT SHOWN IN FIG. 3 AND FIG. 4)

\begin{tabular}{|c|c|}
\hline Parameter & Value \\
\hline $\mathrm{wx}$ & $0.71 \mathrm{~mm}$ \\
\hline $\mathrm{lx}$ & $1.24 \mathrm{~mm}$ \\
\hline wprobe & $0.52 \mathrm{~mm}$ \\
\hline lprobe & $0.50 \mathrm{~mm}$ \\
\hline $\mathrm{x}$ & $0.13 \mathrm{~mm}$ \\
\hline $\mathrm{W}(50 \Omega)$ & $1.11 \mathrm{~mm}$ \\
\hline $\mathrm{hx}$ & $0.88 \mathrm{~mm}$ \\
\hline
\end{tabular}

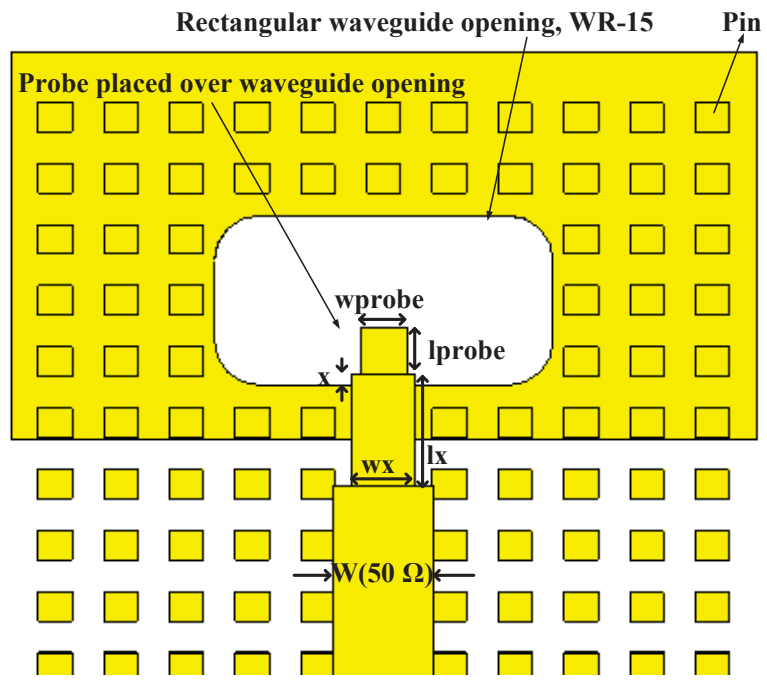

Fig. 3. Transition geometry (top view). Substrate is hidden to allow visualization of rectangular waveguide opening details.

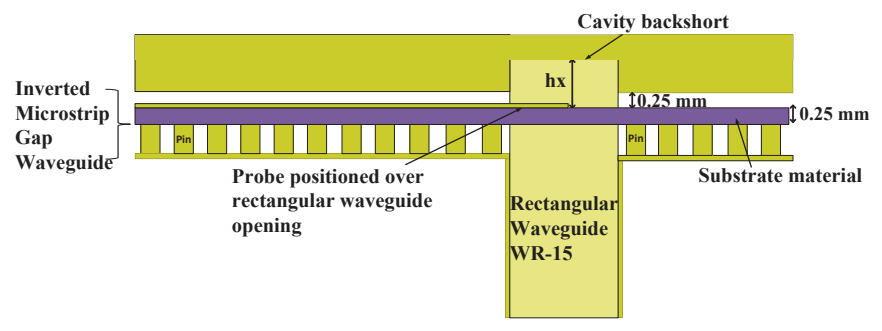

(a)

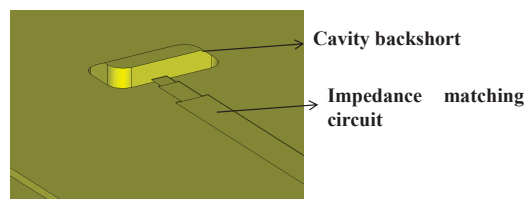

(b)

Fig. 4. (a) Cross-section of complete transition geometry. (b) Cavity backshort placed on top metal lid above PCB and opposite to the rectangular waveguide opening (substrate material is hidden to visualize position of strips with respect to cavity backshort).

numerically analyzed. Afterwards, a $10 \mathrm{~cm}$ (approximately 20 wavelengths) straight inverted microstrip gap waveguide, and another inverted gap prototype that includes two $90^{\circ}$ bends are studied. The second and third prototypes contain

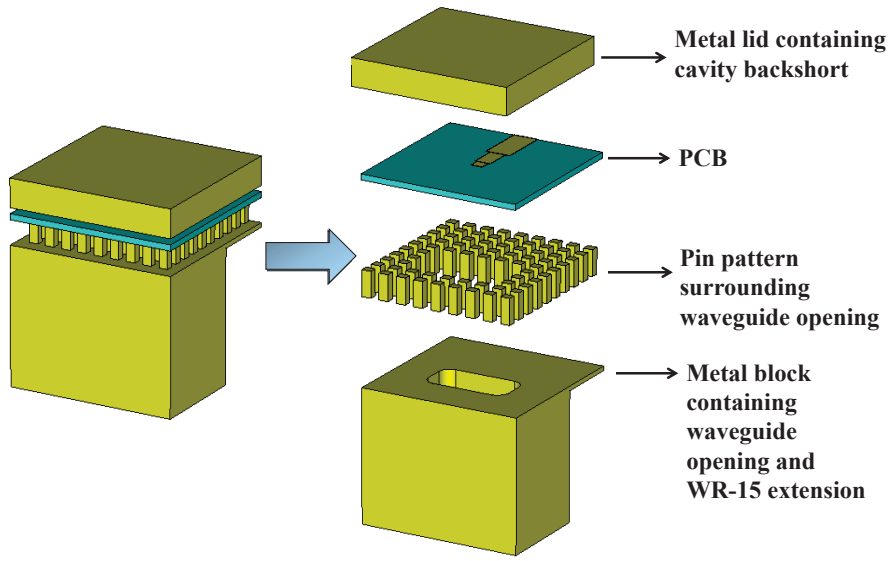

Fig. 5. Simulated single transition from inverted microstrip gap waveguide to rectangular waveguide, with distinction of all different components of the structure.

the transitions proposed in Section III placed at both sides of a $50 \Omega$ feeding line, i.e. constituting two back-to-back transition configurations. The complete analyzed back-to-back geometries are shown in Fig. 6. The simulations of the $\mathrm{S}$ parameters are carried out by using CST Microwave Studio. We should point out that the substrate material employed in our designs is Rogers RO3003 as it was mentioned in Section II. In the Rogers Corporation data sheet, a loss tangent of $\tan \delta=0.0013$ at $10 \mathrm{GHz}$ is given. However, no data of loss tangent as a function of frequency is provided. We did some investigations in order to find further dielectric specifications of Rogers RO3003 material at higher frequencies. In [35] dielectric constant and loss tangent as a function of frequency for different materials are presented. It is stated that the loss tangent of Rogers RO3003 remains below 0.003 between 30 and $67 \mathrm{GHz}$. Thereby, we have considered this data in the simulations of our V-band transitions. The resulted S parameters are illustrated in Fig. 7 for the single configuration case, and Fig. 8 for back-to-back structures.

In the represented $S_{21}$ parameter, the mismatch factor (1$\left|S_{11}\right|^{2}$ ) has been removed in order to just visualize the dissipative contribution of the loss that results from conductive, dielectric and radiation losses. We can also assume that radiation loss is very small since the gap prototypes are properly packaged and any possible radiation due to discontinuities is reduced to a minimum. The expression of dissipation loss is thereby:

$$
\operatorname{Loss}(d B)=10 \log \frac{1-\left|S_{11}\right|^{2}}{\left|S_{21}\right|^{2}}
$$

We should point out that in all the plots shown in this article, the mismatch factor has been removed from the $S_{21}$ parameter in this way.

Simulated results of S parameters for the transition in single configuration (see Fig. 6) show that return loss is larger than $15 \mathrm{~dB}$ from 55 to $70.5 \mathrm{GHz}$, which implies around $24.7 \%$ relative bandwidth. The dissipation loss attributed to a single transition is smaller than $0.5 \mathrm{~dB}$ over the same bandwidth. 


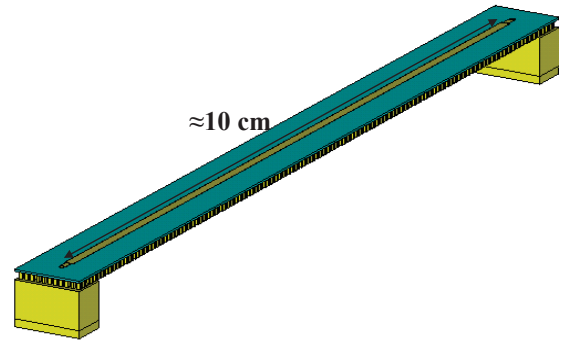

(a)

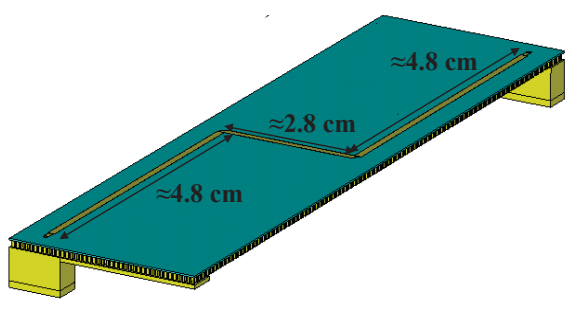

(b)

Fig. 6. Simulated inverted microstrip gap waveguide prototypes (top metal plate containing cavity backshorts is hidden in order to allow visualization of PCB details): (a) Straight $10 \mathrm{~cm}$ line, (b) line with $90^{\circ}$ bends.
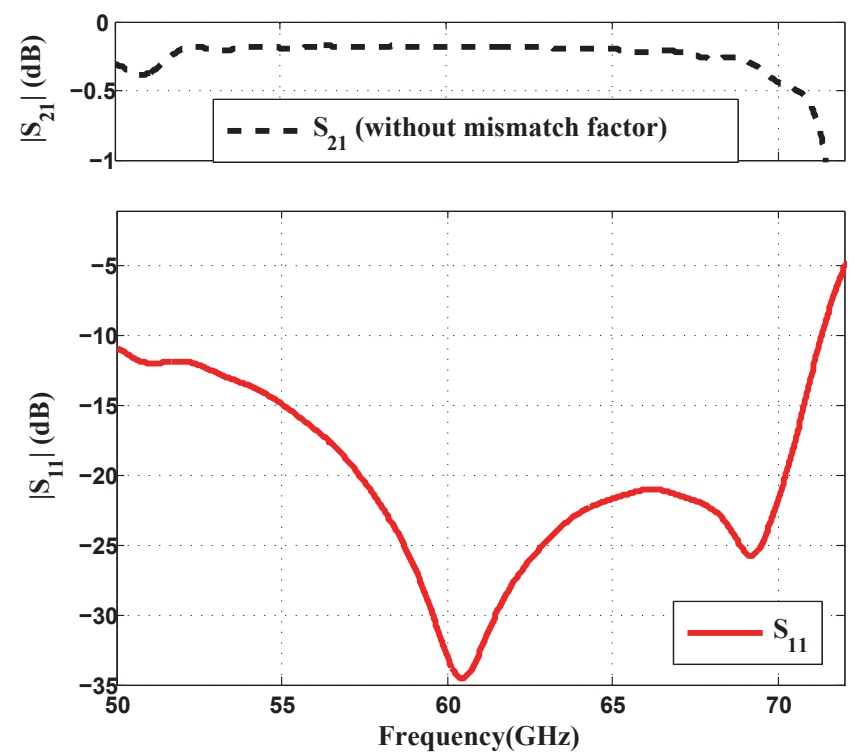

Fig. 7. Simulated $\mathrm{S}$ parameters of a single transition between inverted microstrip gap waveguide and WR-15.

The simulated reflection coefficient of the back-to-back configuration represented in Fig. 8(a), remains below -10 $\mathrm{dB}$ from 53.75 to $70.7 \mathrm{GHz}$ which means $27.2 \%$ relative bandwidth. In the simulated $S$ parameters of the gap prototype with two $90^{\circ}$ bends (see Fig. 8(b)), we can appreciate that return loss is larger than $10 \mathrm{~dB}$ between 54 and $68.5 \mathrm{GHz}$ (i.e. $23.7 \%$ relative bandwidth). The upper limit of the stopband shows up at lower frequency (at around $68.5 \mathrm{GHz}$ ) than in the straight inverted microstrip gap waveguide case. The upper limit of the single-mode frequency band can be identified as the frequency at which resonance peaks start to appear. The dispersion diagram presented in Fig. 2(b) establishes a singlemode band between 52.4 and $68 \mathrm{GHz}$. This dispersion diagram

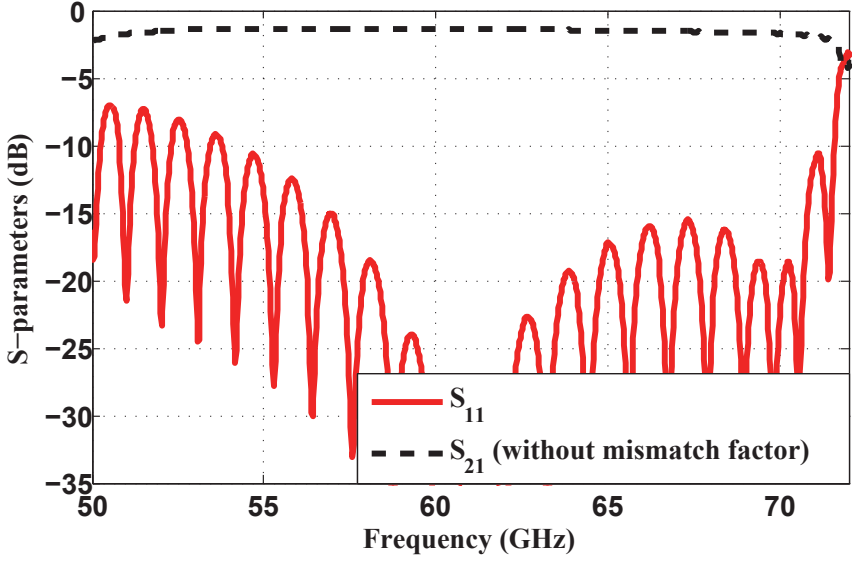

(a)

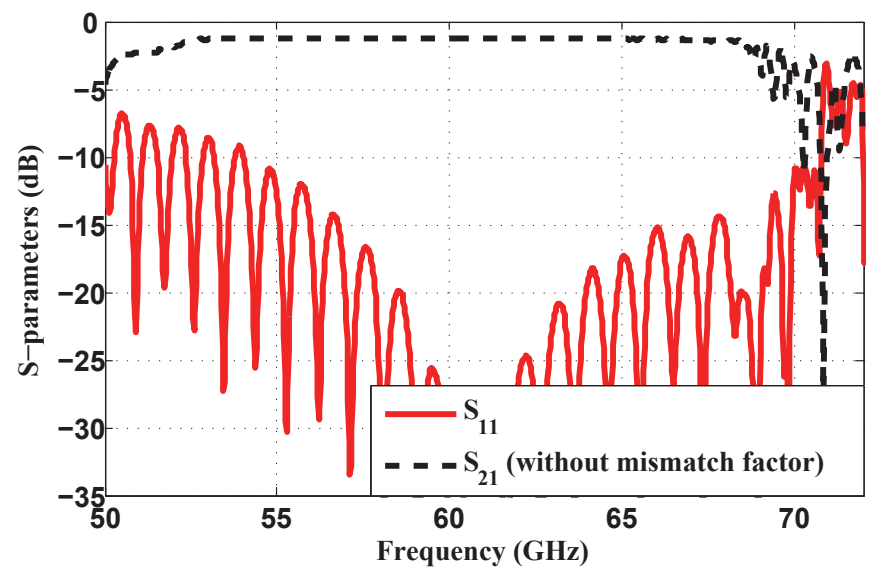

(b)

Fig. 8. Simulated S parameters of two back-to-back configurations, (a) Straight $10 \mathrm{~cm}$ geometry, (b) Prototype with bends.

shows a second strip mode appearing at around $68 \mathrm{GHz}$. This mode shows by further investigations to be asymmetric and causes the ripples of the simulated $S$ parameters of the line with two bends shown in Fig. 8(b). These ripples do not appear in the straight back-to-back line (simulated results presented in Fig.8(a)) because this geometry is symmetric. In spite of this second mode appearing at around $68.5 \mathrm{GHz}$, the transition covers the whole unlicensed $60 \mathrm{GHz}$ band with wide margins.

Dissipation loss for the back-to-back structures is smaller than $1.9 \mathrm{~dB}$ over the previously mentioned bandwidths. The corresponding dissipation loss value at $60 \mathrm{GHz}$ is about 1.3 $\mathrm{dB}$ for both prototypes.

Fig. 9 shows the simulated dissipation coefficient, i.e. transmission coefficient without the contribution due to the mismatch factor, of the $10 \mathrm{~cm}$ straight back-to-back inverted microstrip gap waveguide when considering the combination of different metal and dielectric materials (lossless or lossy materials). The purpose of this study is to examine which is the main contributor to the overall loss of the inverted microstrip gap waveguide. As we can appreciate in Fig. 9, the best case occurs when the conductor material is set as Perfect Electric Conductor (PEC) and the dielectric material is chosen as lossless Rogers RO3003, i.e. the loss tangent is 


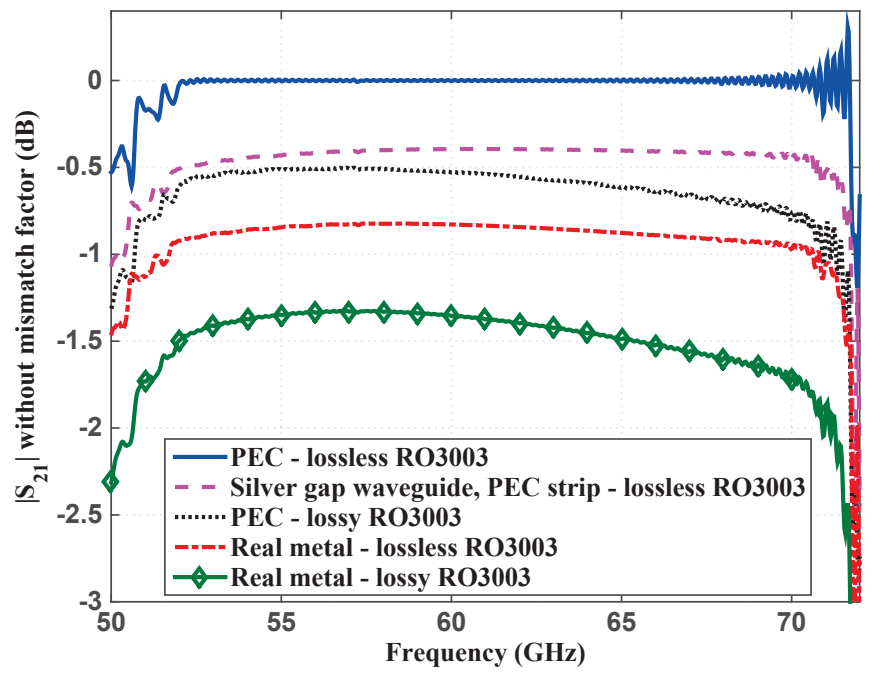

Fig. 9. Transmission coefficient without mismatch factor contribution of a $10 \mathrm{~cm}$ long inverted microstrip gap waveguide (it includes transitions at both sides of the line)

modelled as zero. When the gap waveguide layer is modelled as real silver, and the metal strips of the PCB remain as PEC and the dielectric is lossless, the loss increases around $0.4 \mathrm{~dB}$ at $60 \mathrm{GHz}$. If all metallic parts are real (gap layers are silver and metal strips on the PCB are modelled as copper) and the dielectric material is still considered as lossless, the loss increases by an additional $0.43 \mathrm{~dB}$ at $60 \mathrm{GHz}$. The total loss in the previous situation is larger than if we consider PEC and lossy Rogers RO3003 (setting the real value of loss tangent) where the increase in the dissipation loss with respect to the ideal case is around $0.53 \mathrm{~dB}$. Therefore, we can conclude that the main contributor to the losses of the inverted microstrip gap waveguide is the conductive loss.

\section{Measured Results}

In order to experimentally validate the transition geometry introduced in Section III, two back-to-back structures as the ones shown in Section IV have been manufactured and measured. The mechanical drawings of the straight inverted microstrip gap waveguide are presented in Fig. 10. These drawings are analogous for the prototype with bends. The fabrication of the pin patterns to compose the gap waveguide interface has been realized by using metallic posts created by a Computer Numerical Control (CNC) milling machine. The rectangular waveguide openings needed to fix the WR-15 flanges on the measurement setup, and cavity backshorts are milled out from the lower and upper metal plates respectively. The different building metal blocks are fabricated on brass and silver plated afterwards. On the other hand, the PCB is manufactured by standard etching. The ground plane of the PCB is removed and all metal strips are made of copper. The mechanical assembling of the complete structure is done by using two guiding pins and six screws. Therefore, the prototypes are easy to assemble and disassemble, and no extra gluing or soldering is needed to hold all the different parts of

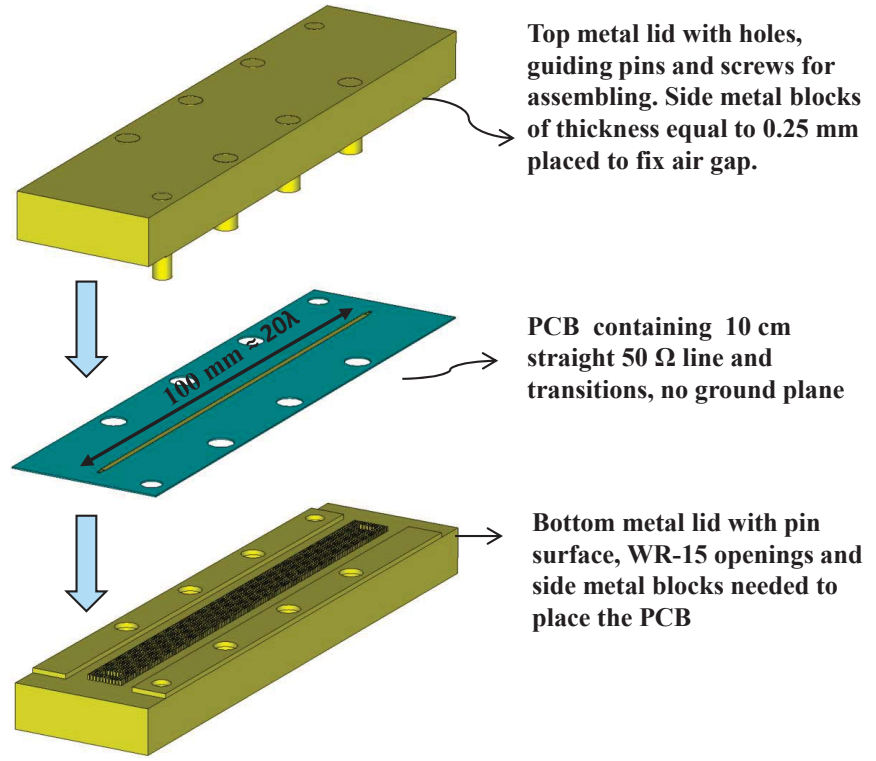

Fig. 10. Mechanical drawings of the manufactured gap waveguide prototypes including back-to-back transitions.

the geometry together. The required air gap is established by adding two metal rectangular steps of thickness equal to 0.25 $\mathrm{mm}$ on the upper metal layer.

Measurements were realized by employing a HP8510C Vector Network Analyzer (VNA) and two V band HP V85104A test set modules. A standard Thru-Reflect-Line (TRL) calibration was performed in order to extract the effect of the connectors and be able to move the measurement reference plane to the WR-15 opening created in the lower metal plate of the fabricated gap waveguides. Pictures of the measurement set up and the two manufactured prototypes are shown in Fig. 11(a), Fig. 11(b) and Fig. 11(c) respectively.

Fig. 12 presents a comparison between simulations and measurements of $\mathrm{S}$ parameters for the straight $10 \mathrm{~cm}$ long inverted microstrip gap waveguide. We can observe that there exists a big shift in frequency of the transition performance (around 7.5 $\mathrm{GHz}$ at lower frequencies and $2.5 \mathrm{GHz}$ at higher frequencies). Measured return loss is about $5 \mathrm{~dB}$ worse in most of the band with respect to simulations, but there is good agreement regarding dissipation loss (see zoomed S21 response in Fig. 13) showing only around $0.17 \mathrm{~dB}$ of discrepancy that can be originated from mechanical and assembling tolerances. These measurement results show that dissipation loss is smaller than $2 \mathrm{~dB}$ over $19 \%$ relative bandwidth, whilst return loss is better than $10 \mathrm{~dB}$ over the mentioned bandwidth.

The shift in frequency and the degradation of the transition performance are limitations that need to be explained. After some investigations in terms of manufacturing and assembling tolerances, we found that the substrate material (Rogers RO3003) is mechanically too soft and therefore bends easily. A consequence of this is that the PCB does not remain rigidly supported over the bed of pins, and there are some points in which the pins do not have a good contact with the substrate (see sketch presented in Fig. 14). We verified by 


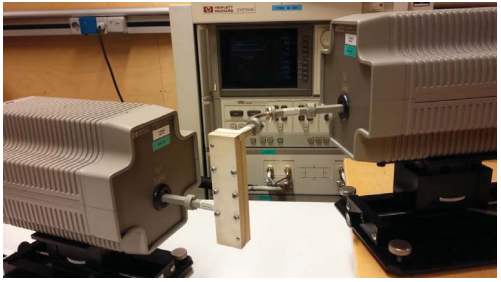

(a)

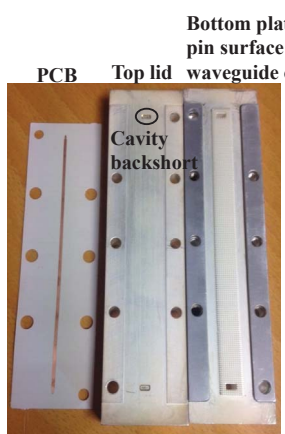

(b)

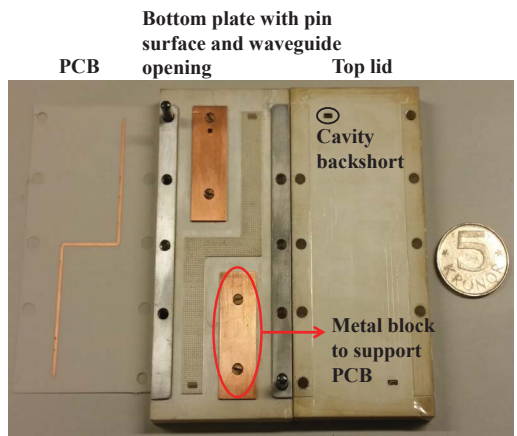

(c)

Fig. 11. (a) Measurement equipment, (b) $10 \mathrm{~cm}$ straight inverted microstrip gap waveguide prototype, (c) Inverted microstrip gap waveguide with bends.

simulations that such non-touching pins become electrically shorter, and thereby the stopband in Fig. 2 shifts towards higher frequencies. As a result, unwanted parallel-plate modes appear at frequencies where the transition is supposed to work well according to our simulated results. This "noncontact effect" between pins and PCB will be demonstrated by simulations in Section VI.

This effect constitutes a critical limitation for the inverted microstrip gap waveguide technology, but different alternative solutions can help to mitigate it. One option is to employ

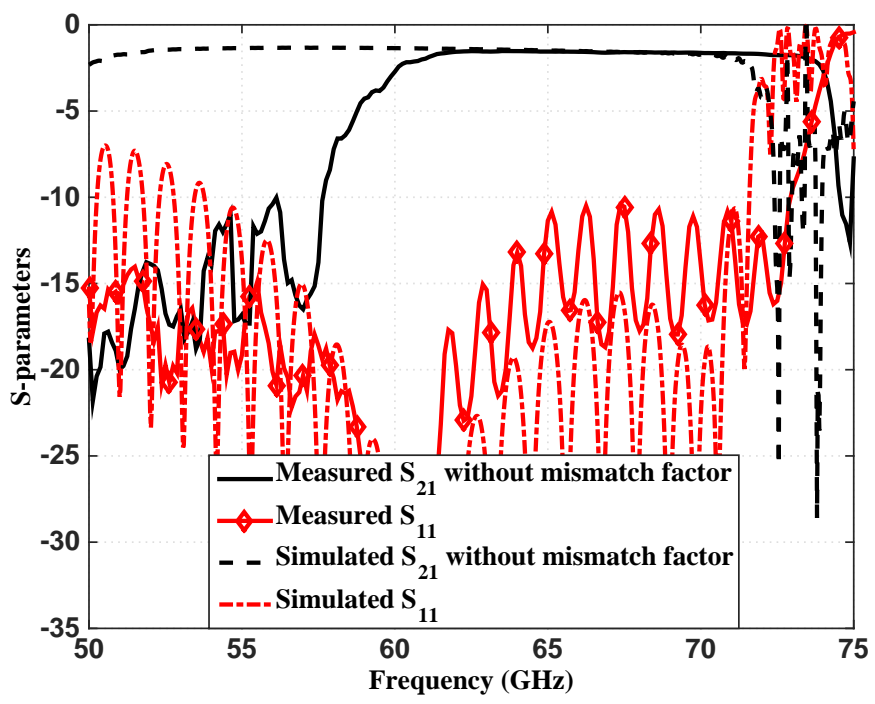

Fig. 12. Simulated and measured S parameters of back-to-back transition between inverted microstrip gap waveguide and WR-15 (straight gap waveguide prototype by using Rogers RO3003 as substrate material).

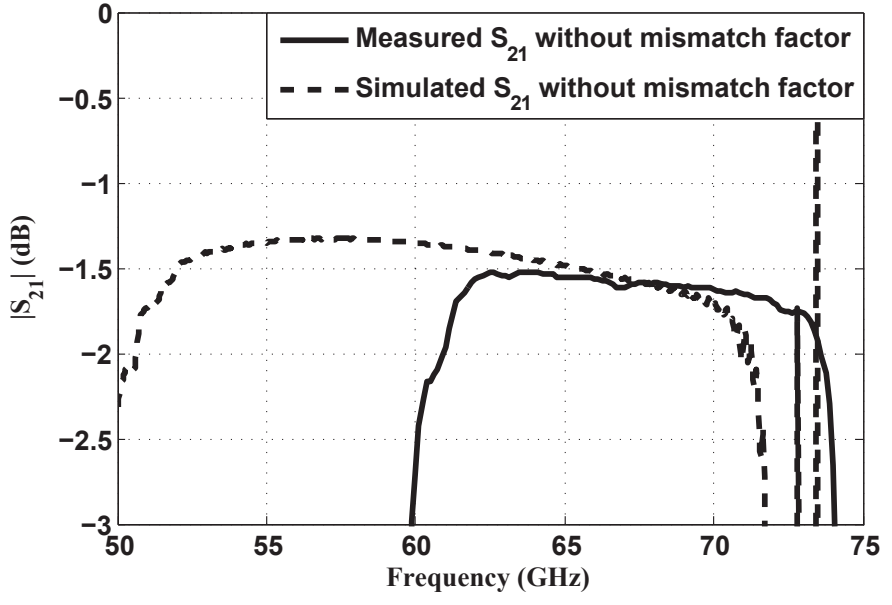

Fig. 13. Zoomed view of simulated and measured $S_{21}$ (straight gap waveguide prototype with Rogers RO3003 as substrate material).

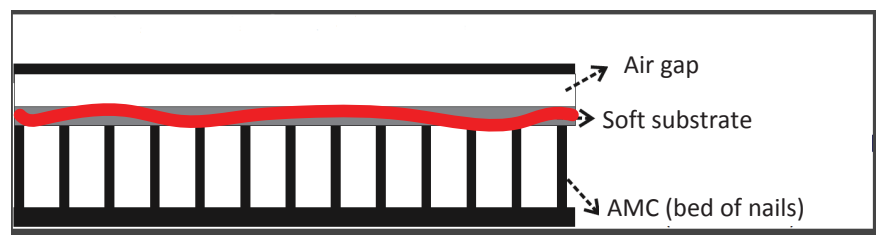

Fig. 14. Sketch of inverted microstrip gap waveguide showing a curved dielectric profile.

more rigid dielectrics such as Rogers RO4003, at the expense of some additional loss. A second alternative is to fill up the gap with thin foam material in order to push the PCB down and thereby ensure good contact with the pins. However, the foam may add loss as well, and we found that foam suppliers do not provide dielectric specifications for millimeter-wave frequencies. Another solution would be to estimate the noncontact effect in advance and design the inverted microstrip gap waveguide with longer pins. In this way we will still get a shift in frequency but we will cover the desired frequency band. We have performed new measurements of the prototypes by considering the first two alternatives.

Firstly, a thin Rohacell HERO71 foam of thickness equal to $0.22 \mathrm{~mm}$ is tested to fill the air gap. Dielectric properties of this foam are not available, so we assume that it is similar to Rohacell HF71 since the chemical natures of HF and HERO are the same according to the supplier. The permittivity is $\epsilon_{r}=$ 1.093 and loss tangent $\tan \delta=0.0155$, both specified at 26.5 $\mathrm{GHz}$ according to the data sheet of HF71 foam. We performed new measurements by filling the gap with the mentioned foam. Fig. 15(a) shows how the foam is placed in the top metal lid. There is an area that has been cut out from the foam in order to allow the fields to propagate within air and reduce the loss attributed to the foam. In this way, the foam still contributes to push down the PCB and ensure better contact between the substrate and the pins, and moreover the additional loss is reduced. A comparison between new simulations and measurements is illustrated in Fig. 15b. The new measured reflection 


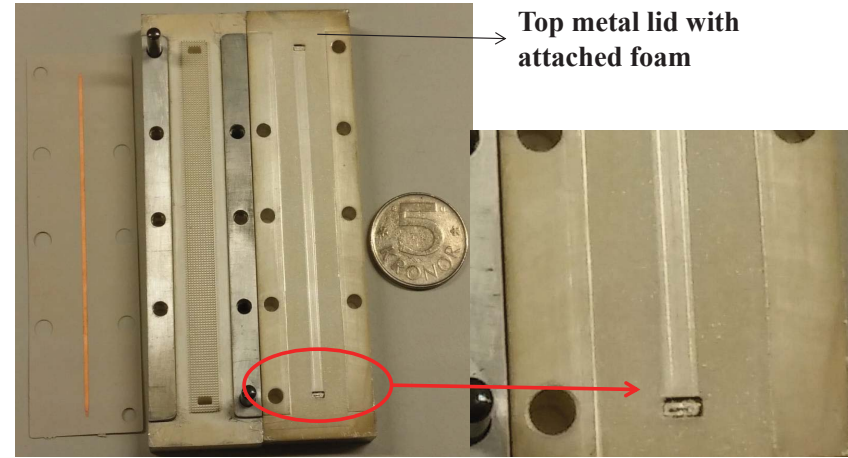

(a)

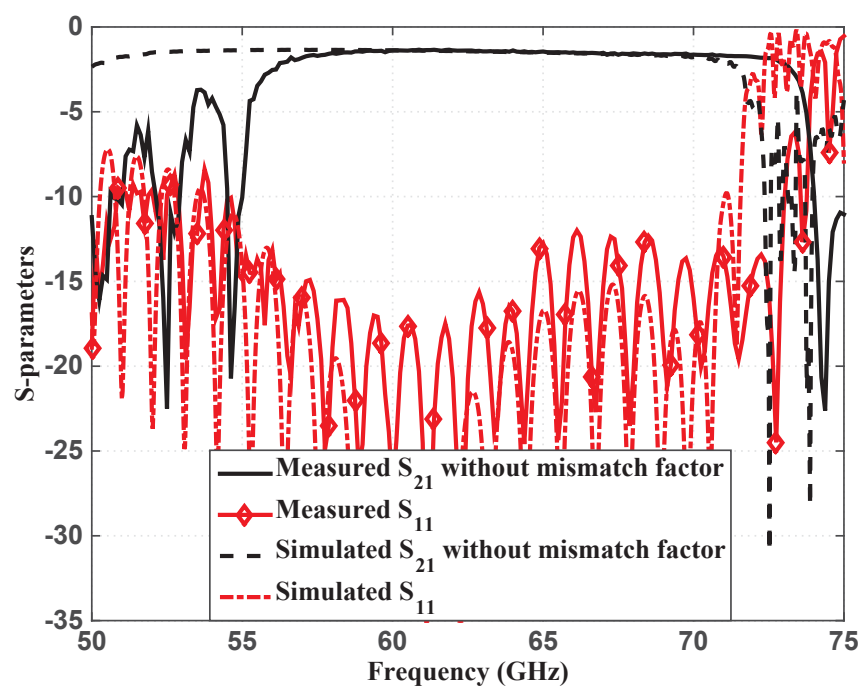

(b)

Fig. 15. (a) $10 \mathrm{~cm}$ straight inverted microstrip gap waveguide prototype with foam attached to the top metal lid (zoomed area shows the section cut out from the foam), (b) Simulations vs measurements of S parameters of back-toback straight inverted microstrip gap waveguide to WR-15 transition (Rogers RO3003 as substrate and Rohacell HERO71 foam attached to top metal lid).

coefficient is lower than $-10 \mathrm{~dB}$ and the experimental dissipation loss is better than $2 \mathrm{~dB}$ from 56.6 to $72.8 \mathrm{GHz}$, which implies $25 \%$ bandwidth. These results are better than the ones we got without using foam, proving that the non-contact effect between pins and PCB is the reason for the discrepancies between measurements and simulations. Moreover, there is good agreement between the measured and simulated insertion loss as seen in Fig. 16. The dissipation factor at $60 \mathrm{GHz}$ is $-1.38 \mathrm{~dB}$ for both simulations and measurements.

Even though these new experimental results are better than the plotted ones in Fig. 12, there is still a shift in the frequency band. Since the structure is very long $(10 \mathrm{~cm})$ it might be difficult to ensure a uniform contact between the bed of pins and PCB in all the points along the geometry. Furthermore, the foam that we use is $0.22 \mathrm{~mm}$ thick since there were no available foams of exactly $0.25 \mathrm{~mm}$, which is the size of the gap. All this can provoke that we do not yet get perfect contact everywhere. Nevertheless, we still observe an important improvement in the measured $\mathrm{S}$ parameters.

Another way to reduce the non-contact effect between pins and PCB is to replace the soft Rogers RO3003 material

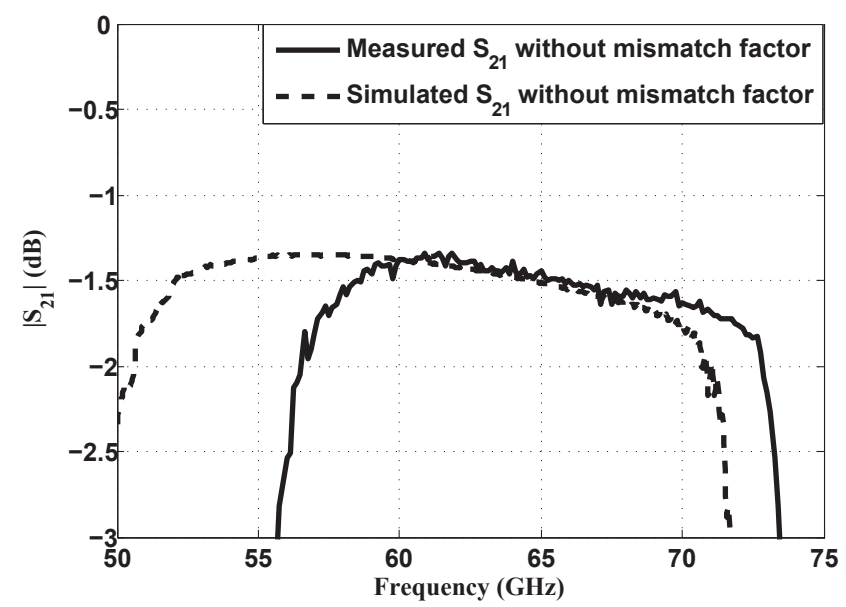

Fig. 16. Zoomed view of simulated and measured $S_{21}$ (straight gap waveguide prototype using Rogers RO3003 as substrate and foam material to fill the gap).

by a more rigid substrate. We have chosen Rogers RO4003 material with permittivity $\epsilon_{r}=3.55$, loss tangent $\tan \delta=0.0027$ (values are specified at $10 \mathrm{GHz}$ ) and thickness $\mathrm{h}=0.203 \mathrm{~mm}$. With these values, the dispersion diagram is very similar to that obtained in Fig. 2. Furthermore, since the fields of the inverted microstrip gap waveguide are mainly confined within the air gap, the fact of using a new substrate with similar permittivity and thickness does not affect the dimensions of the circuit. Thereby, the performance of the transition will be the same without redesigning it (the insertion loss will be a bit higher though due to the new value of the loss tangent). New PCBs were manufactured on Rogers RO4003 and tested re-using the same gap waveguide layers. Measurements and simulations of S parameters are plotted together on Fig. 17, as well as a zoomed version of simulated and measured dissipation factors shown in Fig. 18. First of all, we observe a flat frequency response of the $S_{21}$ parameter over a wider frequency bandwidth than the results presented in Fig. 12 when Rogers RO3003 was the selected substrate. However, there is about $1.4 \mathrm{~dB}$ discrepancy between simulated and measured dissipation losses. The main reason for this may be that our simulations take into account a loss tangent value for Rogers RO4003 specified by the data sheet at $10 \mathrm{GHz}$ since no available data has been found at $60 \mathrm{GHz}$. Therefore, this comparison is not perfectly realistic.

The measured dissipation loss is better than $3 \mathrm{~dB}$ over a $22.65 \%$ relative bandwidth (between 58.25 and $73.13 \mathrm{GHz}$ ). The reflection coefficient remains below $-10 \mathrm{~dB}$ over the same frequency band. These values make clear that using a more rigid material instead of RO3003, improves the circuit performance in terms of bandwidth but with a consequent increase in the loss (Rogers RO4003 has higher loss tangent than RO3003).

As an additional part of this transition study, we also tried to improve the results of Fig. 17 by filling the gap with Rohacell HERO71 foam when using RO4003 substrate. These new measurements are depicted in Fig. 19. The plots of 


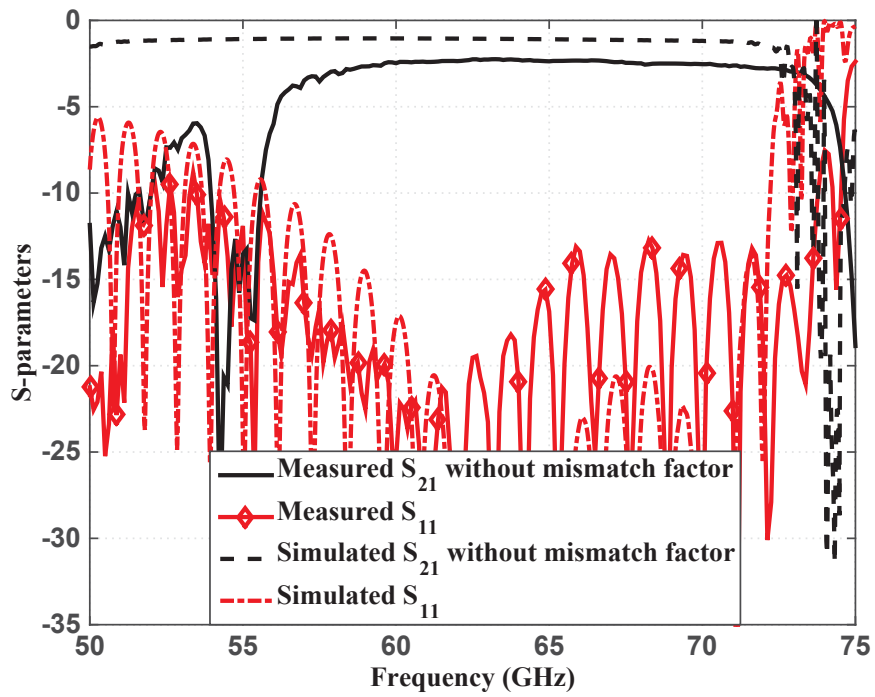

Fig. 17. Simulated and measured S parameters of back-to-back transition between straight inverted microstrip gap waveguide and WR-15 by using Rogers RO4003 as substrate material.

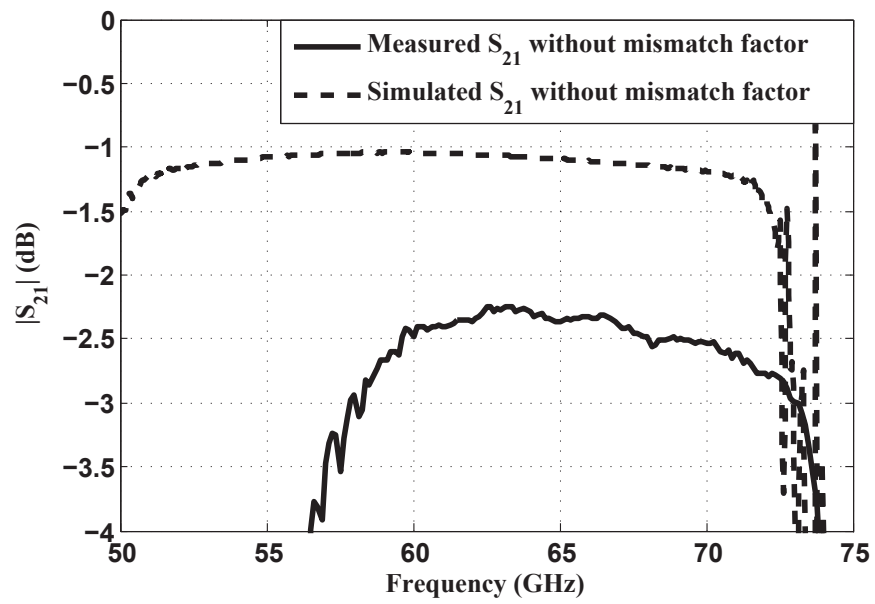

Fig. 18. Zoomed view of simulated and measured $S_{21}$ (straight gap waveguide prototype using Rogers RO4003 as substrate material).

both measured return loss and dissipation loss show improved performance compared to Fig. 17 and Fig. 18 where no foam was added. Fig. 20 illustrates a comparison of simulated versus measured results for the $S_{21}$ parameter and it is clear that the better contact between pins and PCB, the lower losses and larger operating bandwidth. Dissipation losses are lower than $3 \mathrm{~dB}$ from 56.1 to $73.3 \mathrm{GHz}$ which means $26.6 \%$ bandwidth. There is again certain discrepancy between simulated and measured losses. The explanation is again the fact that we do not simulate the structure with the real loss tangent that RO4003 should have at $60 \mathrm{GHz}$. Return loss level remains above $10 \mathrm{~dB}$ over all the previously specified bandwidths.

The last part of our study was to measure an inverted microstrip gap waveguide prototype containing two $90^{\circ}$ bends with Rogers RO3003 substrate and filling the air gap with Rohacell HERO71 foam. We can predict in advance that the

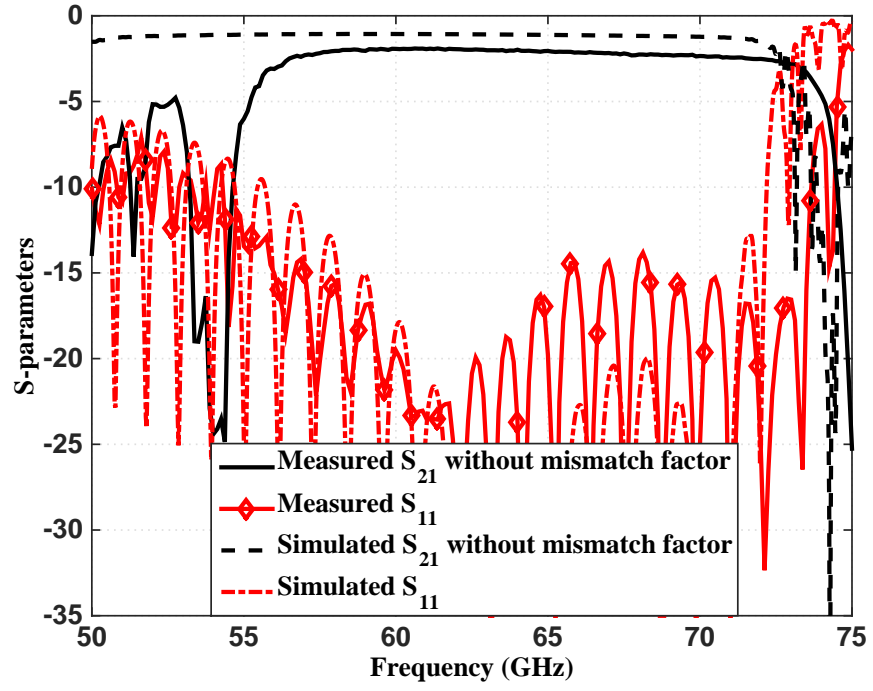

Fig. 19. Simulated and measured S parameters of back-to-back transition between straight inverted microstrip gap waveguide and WR-15 by using Rogers RO4003 as substrate material and Rohacell HERO71 foam to fill the gap.

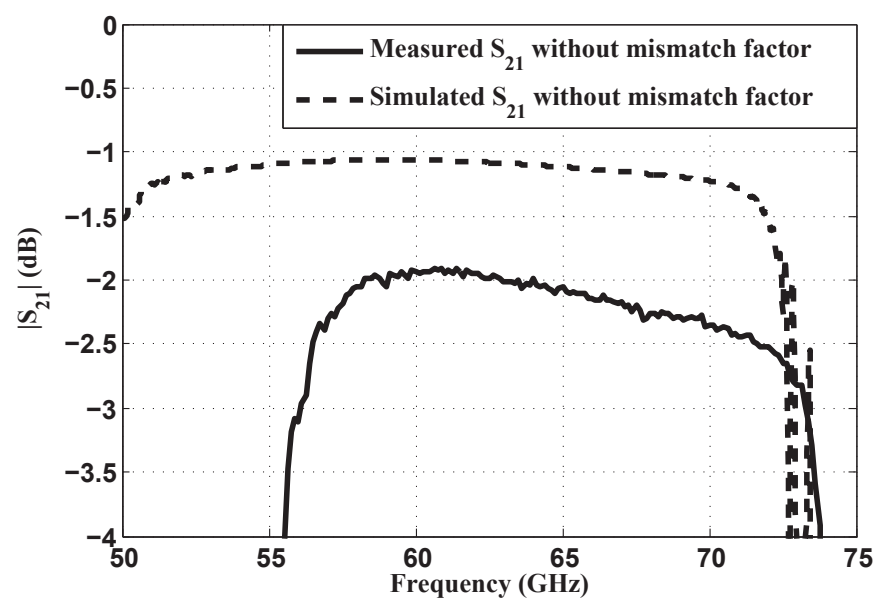

Fig. 20. Zoomed view of simulated and measured $S_{21}$ (straight gap waveguide prototype using Rogers RO4003 as substrate material and Rohacell HERO71 foam to fill the gap).

non-contact effect will be much more critical on this situation for two reasons. The first is that the PCB is wider than in the straight prototype case, so that there will be more points where there is no good contact between pins and dielectric. The second reason is that there is a limited number of rows of only 11 pins each supporting the PCB. This number is more than enough to provide cutoff of fields outside the metal strip that composes the gap waveguide. However, outside the pin area the PCB has no support and bends more easily if we use a soft material such as RO3003. To reduce the consequence of this issue, we added two metal blocks of thickness equal to 1 $\mathrm{mm}$ (the closest to the pin height that we could achieve in the workshop) in the empty areas next to the pin patterns as seen in Fig. 11(c). These metal blocks, together with the foam, 


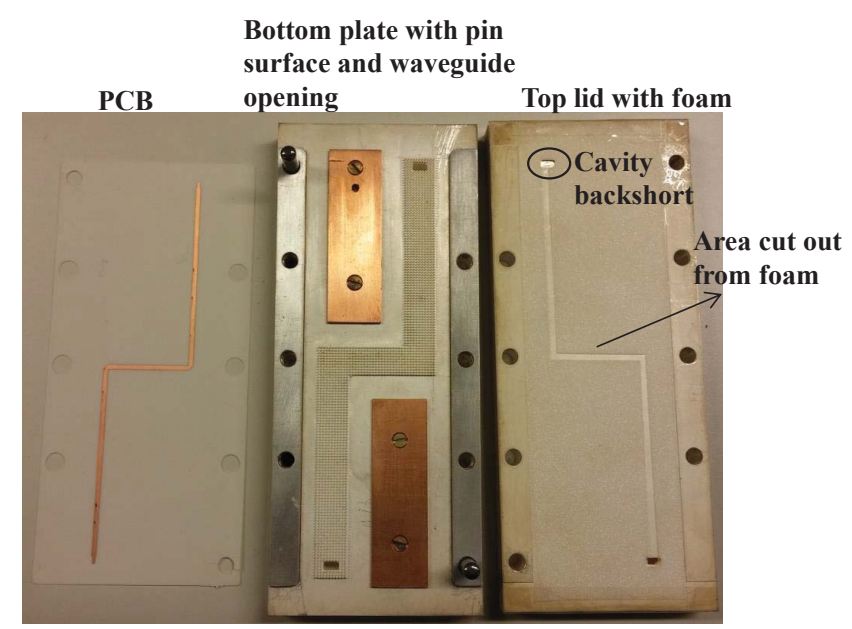

(a)

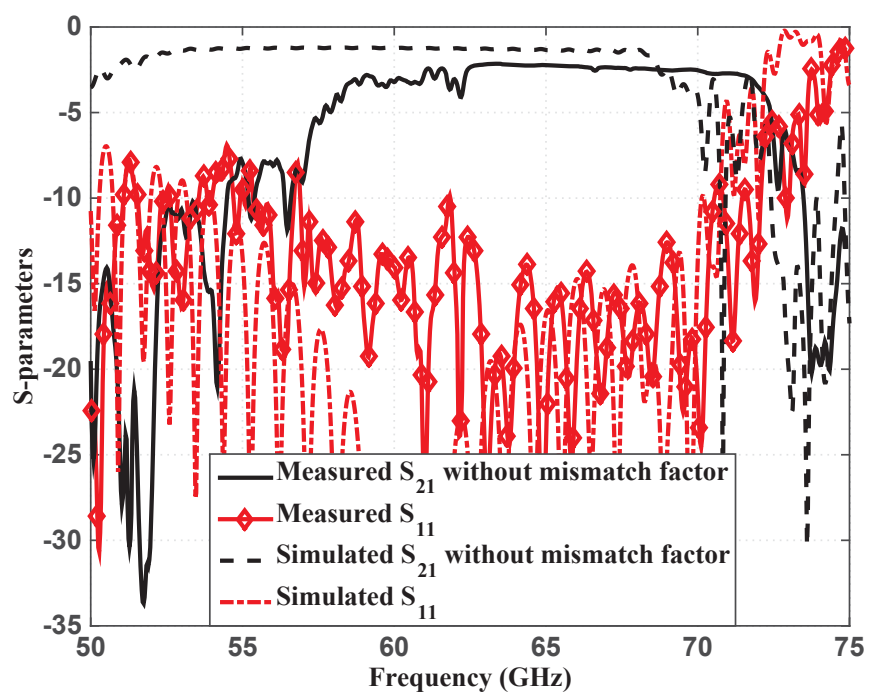

(b)

Fig. 21. (a) Inverted microstrip gap waveguide prototype with bends, foam is attached to top metal lid (zoomed area shows the section cut out from the foam to allow fields to propagate through air), (b) Simulated and measured $\mathrm{S}$ parameters of back-to-back transition between inverted microstrip gap waveguide and WR-15 by using Rogers RO3003 as substrate material and Rohacell HERO71 foam to fill the gap.

are aimed to alleviate the consequences of the non-uniform contact between pins and PCB. Fig. 21(a) shows the inverted microstrip gap waveguide prototype with bends and how the foam is attached into the upper metal lid. There is an area that has been cut out from the foam and has approximately the same shape as the metal strip along which the fields propagate. Fig. 21(b) presents resulting simulated versus measured results of the inverted microstrip gap waveguide with bends including transitions (back-to-back configuration). We observe that there is more discrepancy between simulations and measurements than for the previous situations where we were analyzing a straight geometry. Even by using foam material, there is a large shift in the operating band of the prototype, and there exist some ripples in the lower part of this band.

We can conclude that the wider the structure is, the more critical is the non-contact effect. Moreover, it is very probable
TABLE III

COMPARISON OF MEASURED DISSIPATION LOSS AND OPERATING BANDWIDTH FOR ALL STRAIGHT INVERTED MICROSTRIP GAP WAVEGUIDE PROTOTYPES (INCLUDING BACK-TO-BACK TRANSITIONS TO WR-15) ANALYZED).

\begin{tabular}{|c|c|c|c|c|}
\hline & \multicolumn{2}{|c|}{ RO3003 substrate } & \multicolumn{2}{c|}{ RO4003 substrate } \\
\hline No foam & Foam & No foam & Foam \\
\hline $\begin{array}{c}\text { Best loss value } \\
\text { within } \\
\text { bandwidth }\end{array}$ & $1.53 \mathrm{~dB}$ & $1.34 \mathrm{~dB}$ & $2.25 \mathrm{~dB}$ & $1.91 \mathrm{~dB}$ \\
\hline $\begin{array}{c}\text { Worst loss value } \\
\text { within } \\
\text { bandwidth }\end{array}$ & $2 \mathrm{~dB}$ & $2 \mathrm{~dB}$ & $3 \mathrm{~dB}$ & $3 \mathrm{~dB}$ \\
\hline Operating bandwitdh $^{1}$ & $19 \%$ & $25 \%$ & $22.65 \%$ & $26.6 \%$ \\
\hline
\end{tabular}

${ }^{1}$ Operating bandwidth is defined as the frequency band where return loss is higher than $10 \mathrm{~dB}$ and dissipation loss is better than the specified level as "worst value" on this table.

that the presence of discontinuities combined with the noncontact between pins and PCB at certain points is the main cause for the performance degradations. It would be possible to improve the measured results by employing foams that can fill the gap everywhere to ensure uniform contact. Also, the area that has been cut out from the foam could be realized in a more accurate way in particular around the bends. The best measured dissipation loss is $2.1 \mathrm{~dB}$ at $63.45 \mathrm{GHz}$, and return loss larger than $10 \mathrm{~dB}$ over a $18 \%$ relative bandwidth (from 58.75 to $70.4 \mathrm{GHz}$ ).

Table III summarizes the most important results achieved for the straight inverted microstrip gap waveguide prototypes, including the back-to-back transitions to WR-15 analyzed in this section. From the data of this table we can conclude that by using a more rigid substrate material such as RO4003 and filling at the same time the gap with thin foam, we can reach the best circuit performance (the closest to simulations) in terms of bandwidth and return loss. The drawback is higher insertion loss compared to using Rogers RO3003.

\section{Sensitivity due to Non-CONTACt Between Pins AND PCB}

In this section, we are going to demonstrate by simulations that the deterioration in the circuit performance, as well as the shift in the operating band (both experienced during measurements) are due to the non-contact effect between the bed of pins and the substrate material. For this aim, we generate some curved substrate profiles with a cosine variation along the longitudinal axis, and other profiles with a cosine variation in the transverse direction. In these surfaces, the peak to peak value and the number of periods can be controlled.

Firstly, we consider longitudinal cosine variation, and use a small planar piece of substrate to ensure good contact with the bed of nails at the points where the transitions are operating. There is also contact between the substrate and the bed of nails in those points where the cosine has minima. The microstrip line which is etched on the substrate is generated using the same set of equations. Fig. 22 presents the simulated results including nine different cases, corresponding to one, two and three periods in the cosine variation, for peak to peak excursions of $0.065 \mathrm{~mm}, 0.075 \mathrm{~mm}$ and $0.085 \mathrm{~mm}$. We observe that the performance degrades when there is 


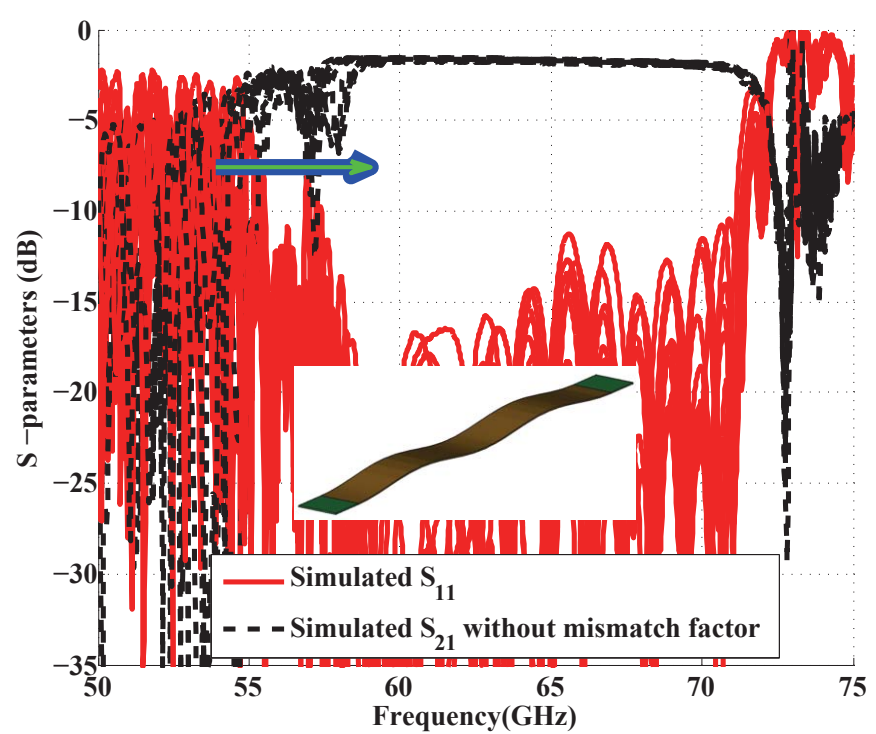

Fig. 22. Simulated S parameters by using generated curved PCB (longitudinal variation and good contact in the transition area).

no uniform contact between pins and substrate. Some low frequency resonant peaks have appeared (indicated with an arrow in Fig. 22). This means that the stopband where the gap waveguide should work has shifted to higher frequencies (around 5-7 GHz depending on the case). Furthermore, there exists a degradation in the $S_{11}$ parameter of approximately 5 $\mathrm{dB}$ with respect to the initial simulations.

Next, we remove the small planar pieces of substrate at the ends of the lines (shown as green flat areas in Fig. 22) and let the whole substrate follow the variation of the cosine function. We consider here two subcases: randomly generated longitudinal cosine variations of the dielectric surface, and variations in the transverse direction as illustrated in the insets

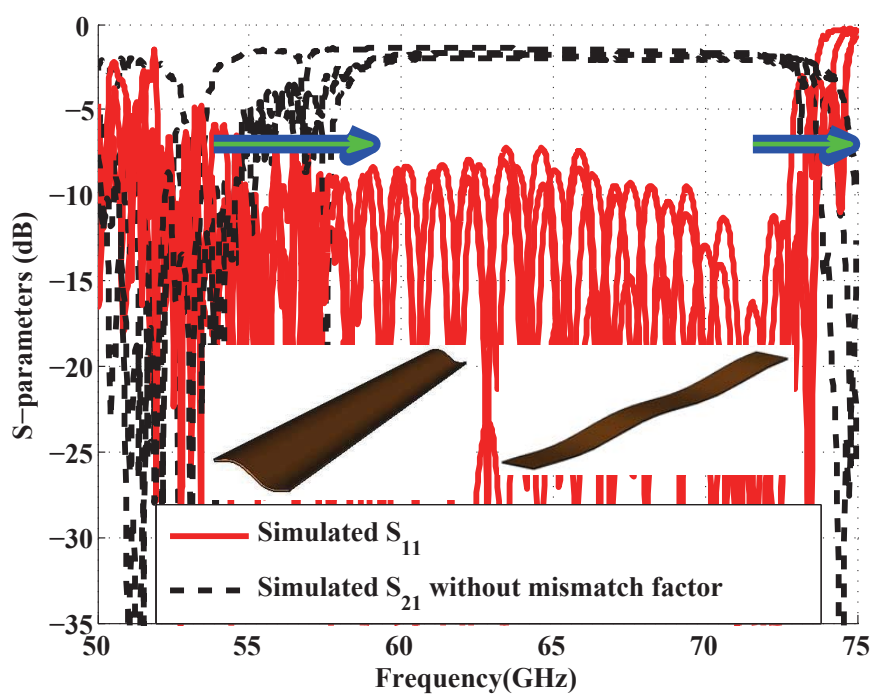

Fig. 23. Simulated S parameters by using generated curved PCB (transversal/longitudinal variation and no good contact in the transition area). of Fig. 23. This figure presents four simulated results for these two cases with a peak-to peak excursion of $0.065 \mathrm{~mm}$, and the obtained effect is the same as that observed in Fig. 22 plus an additional one: the upper frequency limit of the $\mathrm{S}$ parameters moves upwards in the same way as in the measurements. The frequency shift is indicated with arrows in Fig. 23.

These results confirm that the observed frequency shift and degradation of the measured $\mathrm{S}$ parameters are due to the nonuniform contact between the pin surface and the PCB. The experienced shifts in the lower and upper limits of the band are similar to the measured results (around $7.5 \mathrm{GHz}$ in the lower limit and $2 \mathrm{GHz}$ in the upper one). Some other test with two-dimensional randomly generated surfaces with variations in both longitudinal and transverse directions showed similar results.

This effect is a critical limitation for the inverted microstrip gap waveguide technology, but different alternative solutions can mitigate it, which we already explained thoroughly in Section V.

\section{TRANSITION FROM MicROSTRIP PACKAGED BY GAP WAVEGUIDE TO WR-15}

We present here the design of a wideband millimeter-wave transition from standard microstrip (being packaged by gap waveguide technology) to WR-15. The purpose is to compare the losses of the inverted microstrip gap waveguide and a standard microstrip line packaged by gap waveguide.

The previously manufactured gap waveguide layers could be reused, but the PCB was redesigned and assembled to the gap waveguide structure in a different way (see next section).

The cross-section of the complete geometry is shown in Fig. 24(a). We can distinguish two main parts in this structure. A first section composes the gap waveguide layer that contains a pin pattern surrounding the rectangular waveguide opening. This enables connection to rectangular WR-15 waveguide flanges. The second part of the structure consists of a standard microstrip circuit that includes a $50 \Omega$ feeding line terminated by a T-shaped probe which faces the rectangular waveguide opening. A cavity backshort is still needed to be integrated in the upper metal lid. The combination of the T-shaped probe and the cavity backshort ensure field/impedance matching between the local quasi-TEM mode of the microstrip line, and the $T E_{10}$ mode of the rectangular waveguide. The ground plane of the PCB contains a slot that allows the backshort to force the fields to propagate downwards into the waveguide from the microstrip probe. The cavity backshort embedded in the top metal lid and the slot made in the ground plane of the PCB have equal transversal dimensions and are both illustrated in Fig. 24(b).

The layout of the microstrip circuit is depicted in Fig. 25 where the specified parameters are referred to Table IV. The dimensions of the pin surface are the same as shown in Fig. 2 and Table I. Therefore, we can re-use the manufactured gap waveguides for performing measurements of this new transition.

It is important to point out that the air gap now is filled with Rogers RO3003 material for two reasons. The first reason is 

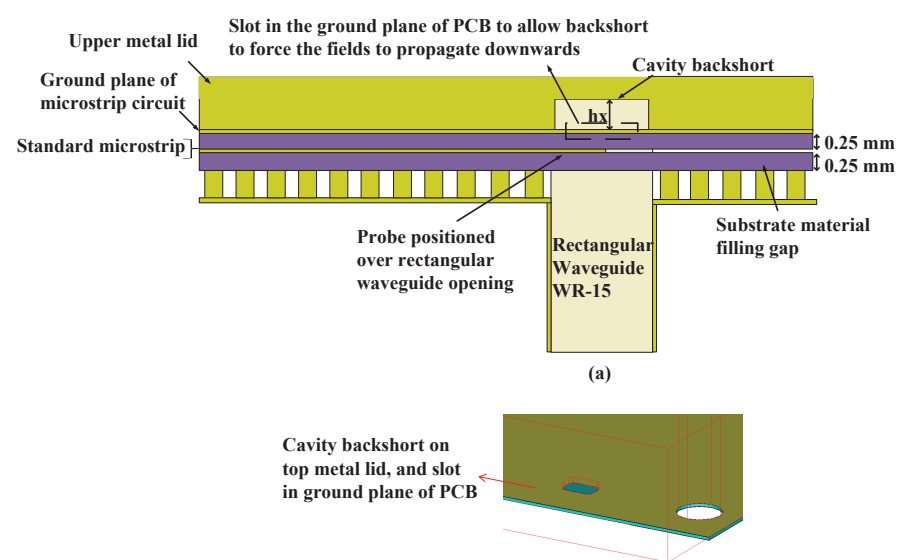

(b)

Fig. 24. (a) Cross-section of complete transition geometry. (b) Cavity backshort placed on top metal lid above PCB and opposite to the rectangular waveguide opening (red lines), and slot made on ground plane of PCB, blue surface is the substrate material under the ground plane.

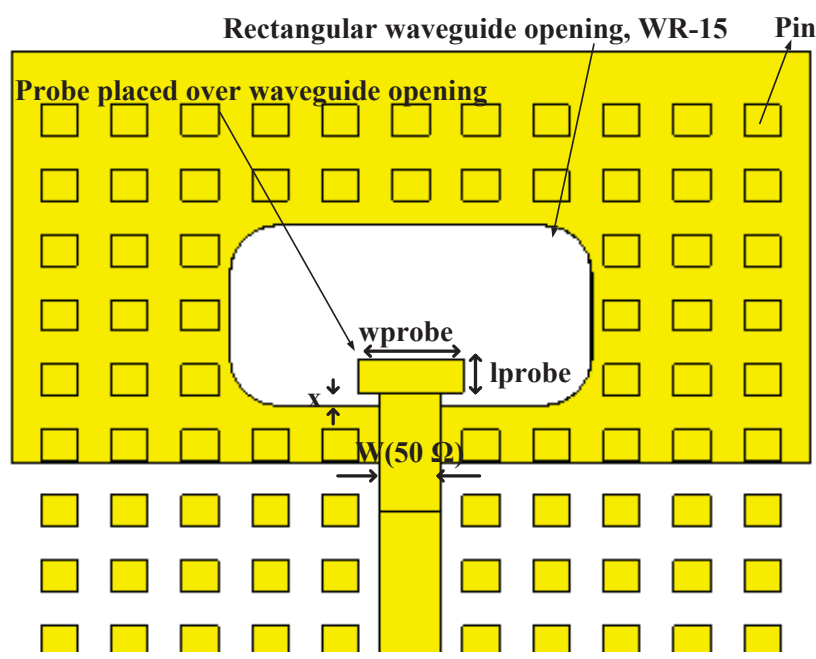

Fig. 25. Transition geometry (top view). Substrate layers and ground plane of PCB are hidden to allow visualization of rectangular waveguide opening details.

TABLE IV

DIMENSIONS OF PCB (REFERRED TO LAYOUT SHOWN IN FIG.24 AND FIG. 25)

\begin{tabular}{|c|c|}
\hline Parameter & Value \\
\hline wprobe & $1.09 \mathrm{~mm}$ \\
\hline lprobe & $0.36 \mathrm{~mm}$ \\
\hline $\mathrm{x}$ & $0.135 \mathrm{~mm}$ \\
\hline $\mathrm{W}(50 \Omega)$ & $0.64 \mathrm{~mm}$ \\
\hline $\mathrm{hx}$ & $0.52 \mathrm{~mm}$ \\
\hline
\end{tabular}

illustrated in Fig. 26. If the gap is filled with air (Fig. 26(a)), the obtained dispersion diagram of the new geometry does not coincide with the $60 \mathrm{GHz}$ band that is covered by the prototypes investigated in Sections II-VI. However, if we fill the gap with Rogers RO3003, the pins are electrically longer and the stopband is shifted to lower frequencies. Thus, it

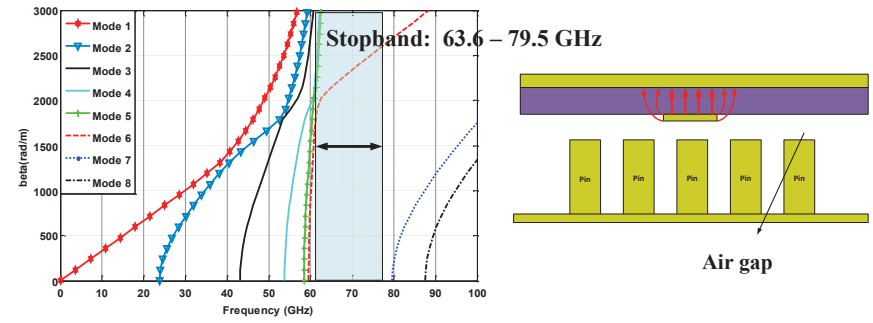

(a)

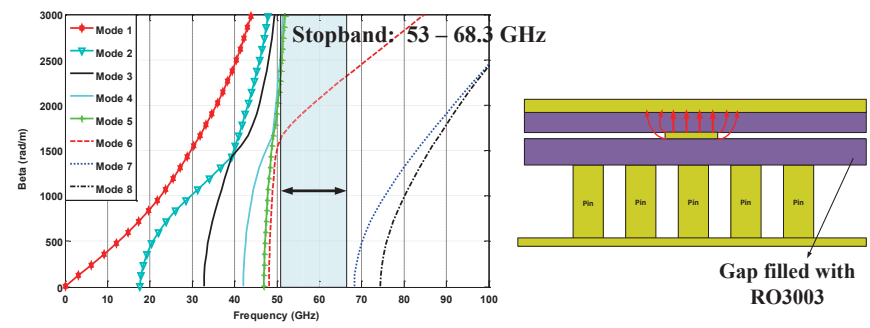

(b)

Fig. 26. (a) Dispersion diagram for the corresponding microstrip geometry when gap is filled with air. (b) Dispersion diagram for the corresponding microstrip geometry when gap is filled with substrate material.

covers the frequency band from 53 to $68.3 \mathrm{GHz}$ (Fig. 26(b)). In this way, we can make a fair loss comparison in the same frequency interval, between the obtained measured results of the inverted microstrip gap waveguide circuits and the new designed microstrip prototypes. The second reason is that we take the advantage of fixing the gap by using a substrate of the same thickness and without the need of making any extra step in the metal blocks. In spite of filling the gap with RO3003, the fields still mainly propagate within the corresponding substrate material of the PCB since the geometry is packaged by gap waveguide and there is cutoff of fields between the pin surface and the metal strip of the PCB.

\section{Simulated vs Measured Results}

The transition design proposed in Section VII is numerically analyzed in terms of S parameters in single and back-to-back configurations. A sketch of the different parts composing the single transition geometry is presented in Fig. 27(a) and the corresponding simulated S parameters are shown in Fig. 27(b). The simulated return loss is larger than $15 \mathrm{~dB}$ over about $28.3 \%$ relative bandwidth (from 50.75 to $67.45 \mathrm{GHz}$ ), while the resulting dissipation loss is smaller than $0.5 \mathrm{~dB}$ over the same bandwidth. The dissipation factor for the presented single transition within the stopband shown in Fig. 26(b) would be smaller than $0.2 \mathrm{~dB}$.

As we said, the manufactured gap waveguide prototypes employed to build the inverted microstrip gap waveguide designs investigated in Sections II-VI, were reused to constitute the standard microstrip lines packaged by gap waveguide technology. The assembling of the different building blocks is illustrated in Fig. 28. Fig. 29(a) shows the measured prototype and Fig. 29(b) presents a comparison between simulations and measurements of two back-to-back transitions connected by a $10 \mathrm{~cm} 50 \Omega$ microstrip line. The plotted parameters show good 


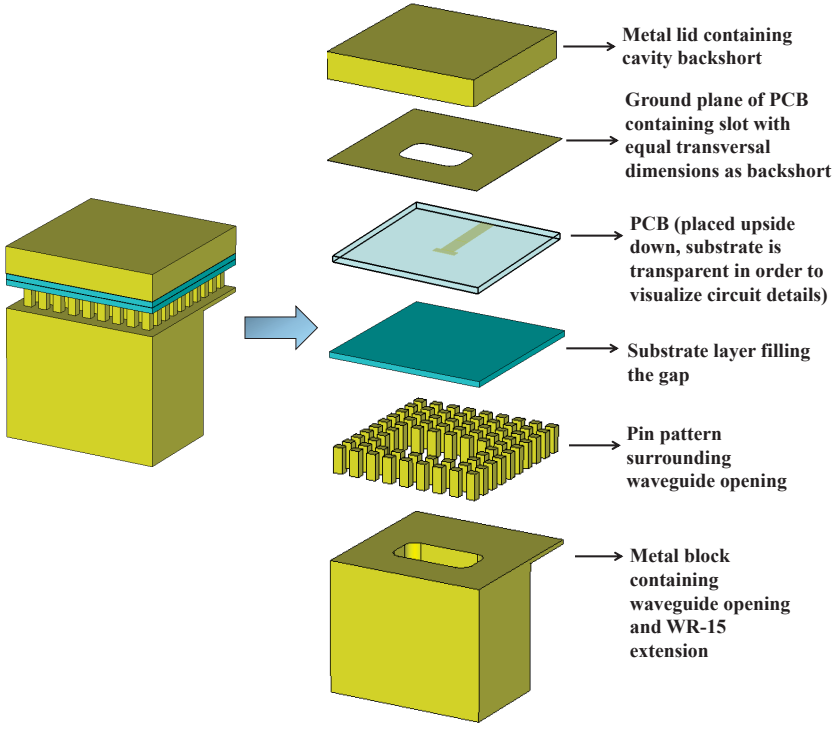

(a)
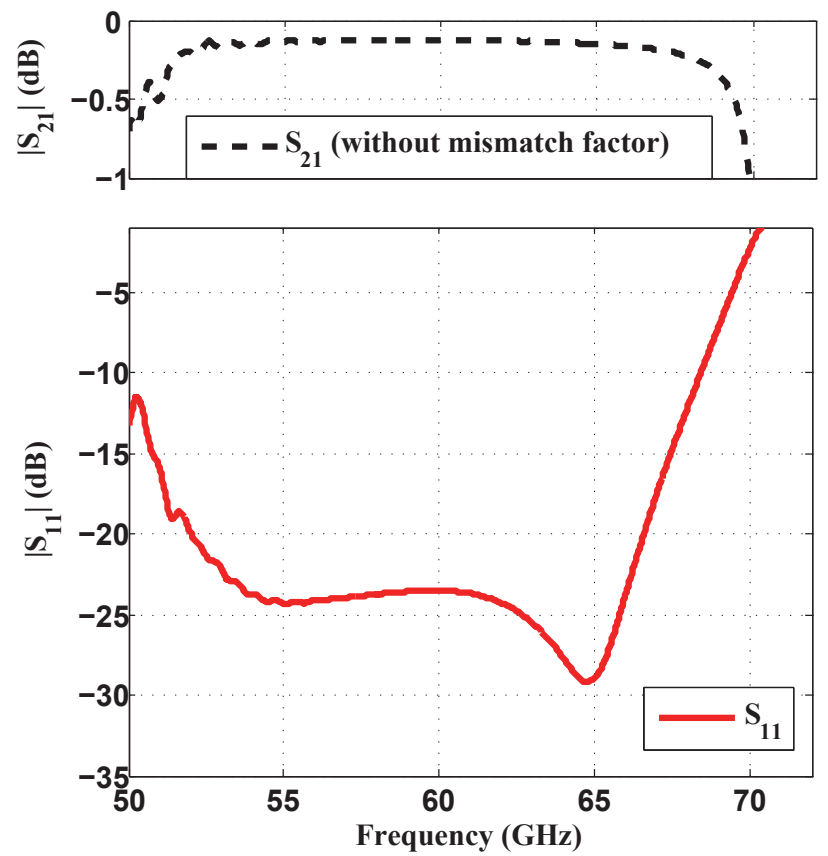

(b)

Fig. 27. (a) Sketch of simulated single transition from standard microstrip packaged by gap waveguide to rectangular waveguide, with distinction of all different components of the structure. (b) Simulated S parameters of a single transition between standard microstrip packaged by gap waveguide and WR15.

agreement between measurements and simulations in terms of losses. However, there is again a shift in the operating band of the measured prototype. This is as before attributed to the non-uniform contact between the pin surface and the substrate layer that fills the gap. Rogers RO3003 material is so soft that it can have a curved profile even when it is used to fill the air-gap. Since the structure is quite long, there might be points where the dielectric layer does not make good contact with the pins, thereby shifting to higher frequencies. Fig. 30 presents a zoomed version of the transmission coefficient showing
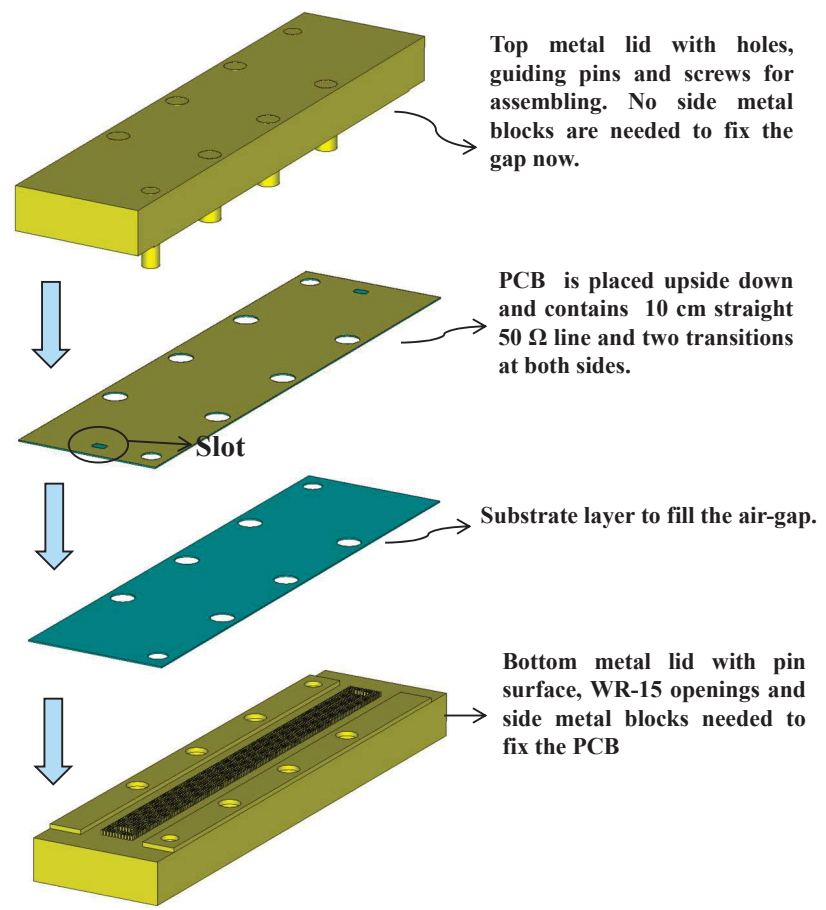

Bottom metal lid with pin surface, WR-15 openings and side metal blocks needed to fix the PCB

Fig. 28. Mechanical drawings of the manufactured gap waveguide prototypes including back-to-back transitions from microstrip to WR-15.

a smaller value than $-5 \mathrm{~dB}$ over about $13.8 \%$ bandwidth (between 58.6 and $67.3 \mathrm{GHz}$ ). The experimental return loss is larger than $15 \mathrm{~dB}$ over the mentioned frequency band.

\section{LOSS COMPARISON}

After the validation of the proposed millimeter-wave backto-back transitions from two different gap waveguide versions to rectangular waveguide, we compare the simulated/measured loss in this section. Four planar back-to-back structures are involved in this comparison: inverted microstrip gap waveguide, standard microstrip packaged by using gap waveguide technology, standard microstrip without including any type of packaging (data obtained from the catalogue of Southwest Microwave, in the corresponding section about end launch connectors), and substrate integrated waveguide (measurements and simulation results are presented in [36]). Table V summarizes the minimum and maximum dissipation loss expressed in $\mathrm{dB} / \lambda_{g}$ over the operating bandwidth (also specified in the table). In all analyzed cases, the mismatch factors have been extracted from the original $S_{21}$ parameters in order to only visualize the dissipative component of the insertion loss and achieve a fair comparison among all prototypes.

The microstrip geometry used in the Southwest Microwave catalogue has the same substrate thickness as the Rogers RO4003 material employed on the design of our inverted microstrip gap waveguide prototypes explained in previous sections. This allows us to realize a straightforward comparison between both structures. We can clearly see that the loss attributed to the unpackaged microstrip is much larger than for the inverted microstrip gap waveguide case. One of the reasons for this is a reduction of the conductive 


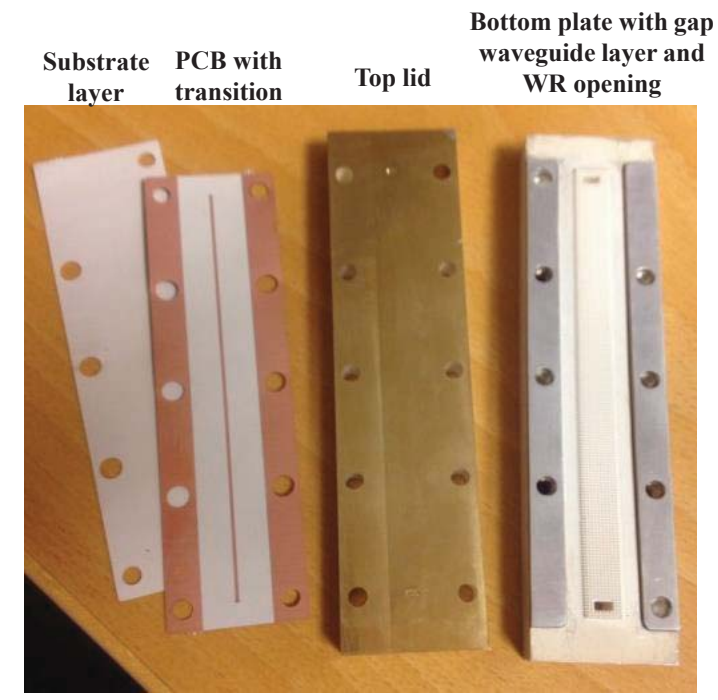

(a)

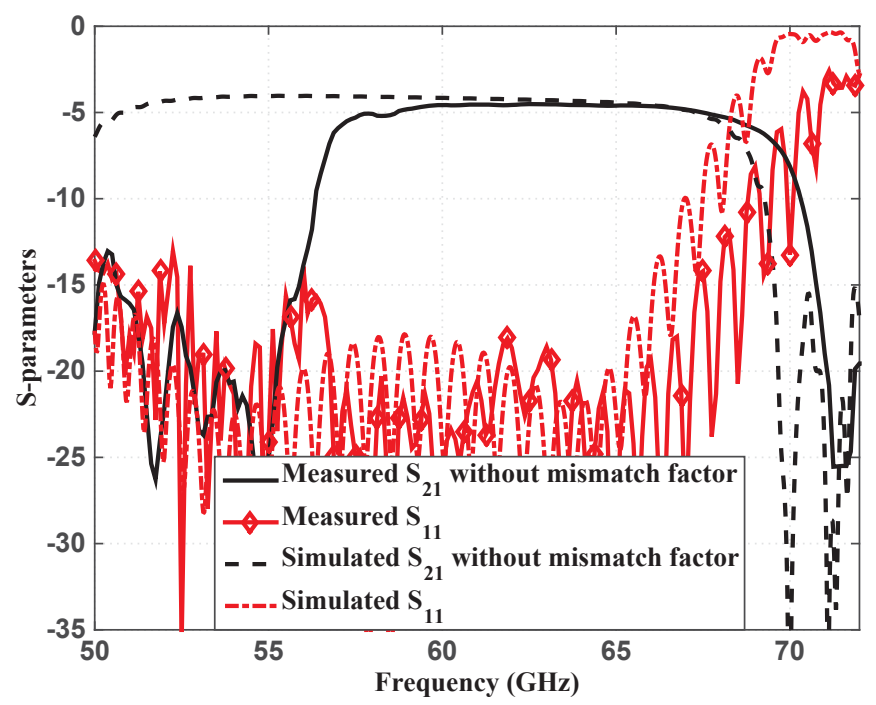

(b)

Fig. 29. (a) Microstrip packaged by gap waveguide, (b) Simulated and measured S parameters of back-to-back transition between standard microstrip packaged by gap waveguide and WR-15 by using Rogers RO3003 as substrate material of PCB and layer to fill the gap.

loss in the inverted microstrip gap waveguide compared to microstrip. This is caused by the fact that for achieving same characteristic impedance, the obtained transversal dimensions of the lines in the inverted microstrip gap waveguide become wider than in the microstrip geometry, hence reducing the conductive loss. On the other hand, the fields are propagating within air in the inverted microstrip gap waveguide geometry, thereby reducing the dielectric loss. Furthermore, since the microstrip line is not properly packaged, the radiation loss is also an important additional contributor to the overall loss. The inverted microstrip gap waveguide is inherently packaged and the radiation loss is non-existent.

When we package a microstrip circuit with gap waveguide technology (as we did in sections VII-VIII), the radiation loss is reduced to zero and the fields are completely confined

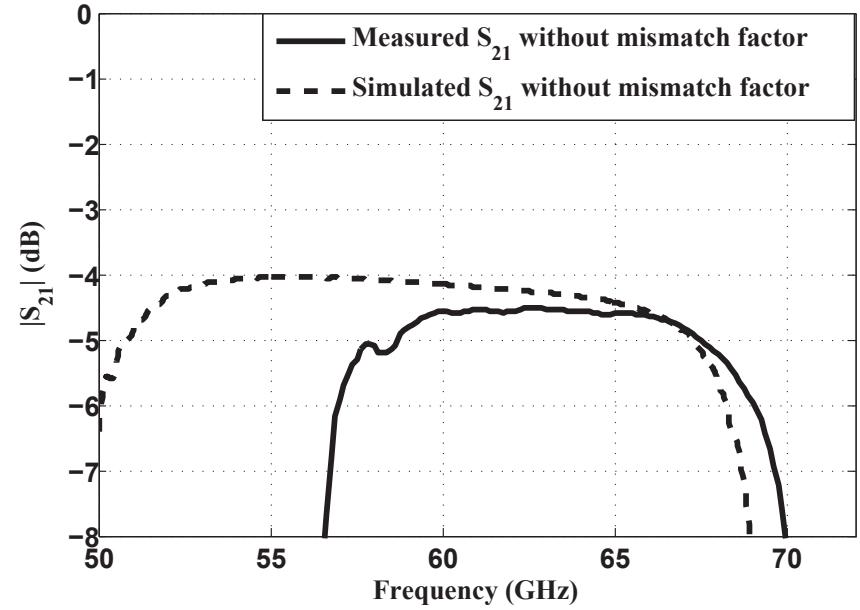

Fig. 30. Zoomed view of simulated and measured $S_{21}$ (back-to-back configuration of transition from standard microstrip packaged by gap waveguide to WR-15).

within the substrate. This confirms that the attenuation for a microstrip packaged with gap waveguide is lower than for an open microstrip geometry as it is mentioned in Table V.

On the other hand, the measured loss of SIW is slightly larger than for the inverted microstrip gap waveguide designed with RO3003, and has about the same loss magnitude compared to the gap prototype with RO4003 material. However, it is important to point out that the SIW geometry shown in [36] has been designed with low loss RT/duroid 5880 material that has a loss tangent close to 0.001 at $60 \mathrm{GHz}$ (see material specifications in [37]). Therefore, if the inverted microstrip gap waveguide would have been designed with such material, the resulted insertion loss would be lower than the obtained for SIW. A CST simulation of our inverted microstrip gap waveguide design considering RT/duroid 5880 (with thickness equal to $0.25 \mathrm{~mm}$ ) has been performed in order to get an approximate idea of the corresponding loss with that material. The simulated results show a minimum loss of $0.045 \mathrm{~dB} / \lambda_{g}$, which constitutes a smaller value than that obtained for SIW case in [36]. We should remark that we are still using the same transition geometry as proposed in Section III. This design could have been re-tuned in order to optimize the gap waveguide circuit performance when using RT/duroid 5880 material, and thus obtaining a better loss value. We have also compared the transition geometry presented in [36] and our inverted microstrip gap waveguide transition design in terms of design complexity and fabrication. In such SIW transition, a multilayer structure is required to achieve a wide operating frequency band. Each layer has via holes with different periods and are placed in a non-uniform way. Therefore, there are many design parameters involved in the structure, that implies a more complex and time-consuming design process. However, in our structure we fix a uniform pattern of pins that we never optimize to attain a good transition performance. The only dimensions we need to optimize are the matching probe sections and the depth of the backshort. Moreover, the SIW transition structure requires via holes to be included around the coupling patch in the upper substrate layer, to avoid potential 
TABLE V

COMPARISON OF DISSIPATION LOSS

\begin{tabular}{|c|c|c|c|}
\hline \multirow{2}{*}{ Prototype } & \multicolumn{2}{|c|}{$\begin{array}{c}\text { Measured } \\
\text { dissipation loss }\end{array}$} & $\begin{array}{c}\text { Simulated } \\
\text { dissipation loss }\end{array}$ \\
\cline { 2 - 4 } & $\begin{array}{c}\text { Min. } \\
\left(\mathrm{dB} / \lambda_{g}\right)\end{array}$ & $\begin{array}{c}\text { Max. } \\
\left(\mathrm{dB} / \lambda_{g}\right)\end{array}$ & Min. (dB/ $\left.\lambda_{g}\right)$ \\
\hline $\begin{array}{c}\text { Inverted microstrip } \\
\text { gap waveguide } \\
\text { (RO3003+foam) } \\
(56.6-72.8 \mathrm{GHz})\end{array}$ & 0.061 & 0.092 & 0.061 \\
\hline $\begin{array}{c}\text { Inverted microstrip } \\
\text { gap waveguide } \\
\text { (RO4003+foam) } \\
(56-73.3 \mathrm{GHz})\end{array}$ & 0.087 & 0.137 & 0.046 \\
\hline $\begin{array}{c}\text { Standard microstrip } \\
\text { packaged with gap } \\
\text { waveguide (RO3003) } \\
(58.6-67.3 \mathrm{GHz})\end{array}$ & 0.129 & 0.142 & 0.115 \\
\hline $\begin{array}{c}\text { Unpackaged } \\
\text { microstrip }{ }^{1}(\mathrm{RO} 4003) \\
(55-67 \mathrm{GHz})\end{array}$ & 0.343 & 0.397 & --- \\
\hline $\begin{array}{c}\text { SIW }{ }^{2} \\
(\mathrm{RT} / \mathrm{duroid} 5880) \\
(55-67 \mathrm{GHz})\end{array}$ & 0.068 & 0.156 & 0.052 \\
\hline
\end{tabular}

${ }^{1}$ Data extracted from catalogue of Southwest Microwave (end launch connectors).

${ }^{2}$ Data obtained from [36]

leakage coming from surface waves. This is not needed in the proposed gap waveguide transitions, since all parallelplate modes and surface waves are suppressed within the stopband. On the other hand, the manufacturing of a uniform pin pattern is quite simple and can be done in techniques suitable for mass production such as micromachining and molding. The manufacturing of the required PCB in the inverted microstrip gap waveguide can be simply done by standard photolithography technique. However, we should remember that for designing inverted microstrip gap waveguides, rigid substrate materials are needed in order to mitigate the noncontact effect between pins and PCB. We should consequently make a trade off when choosing the substrate type considering loss, rigidness and cost.

\section{Conclusions}

Two broadband millimeter-wave gap waveguide transitions have been presented. The first geometry acts as an interface between inverted microstrip gap waveguide and standard WR-15. The performance has been determined by simulations (in both single and back-to-back configurations) and measurements of electrically long (around 20 wavelengths) back-to-back prototypes. The experimental results show $S_{11}$ smaller than $-10 \mathrm{~dB}$ over relative bandwidths larger than $25 \%$ and $26.6 \%$ when Rogers RO3003 and RO4003 are employed, respectively. A shift in the operating band appears in all measurements. This reveals a critical limitation of the inverted microstrip gap waveguide technology. The reason for this frequency shift is the non-uniform contact between the PCB and the pins along the gap waveguide, and it is more critical the larger the PCB is. We have proposed different ways to mitigate this effect, and we carried out a numerical study of the circuit performance when the substrate was curved. The simulations confirm our explanation. In spite of the non-contact effect, the proposed transition covers the whole $60 \mathrm{GHz}$ band and its simple and compact configuration makes it suitable to be integrated into an inverted microstrip gap waveguide feednetwork for slot/horn array antenna applications. There is no requirement for any complex geometry modifications in the waveguide structure itself to ensure a good integration with the transition. We only need a waveguide opening milled out in the lower metal plate to attach the rectangular waveguide flange.

The second design consists of a transition from standard microstrip packaged by a bed of pins to WR- 15 . The performance was determined by simulating it in single and back-toback configuration, and by measuring back-to-back prototypes. There is good agreement between simulated and measured results. However, there is a frequency shift in the performance, caused by the substrate material (Rogers RO3003) filling the gap, being so soft that again it gave non-uniform contact to the pins. The measured return loss is still larger than $15 \mathrm{~dB}$ over $13.8 \%$ relative bandwidth.

Finally, we have studied the losses of the two investigated gap waveguide types in the $60 \mathrm{GHz}$ band, and compared them with unpackaged microstrip and substrate integrated waveguide. Unpackaged microstrip presents the largest attenuation, caused by dielectric, conductive and radiation loss. Inverted microstrip gap waveguide with Rogers $\mathrm{RO} 3003$ material has lowest measured losses. The obtained loss of the inverted microstrip gap waveguide with Rogers RO4003 is slightly larger than when using RO3003, and about the same as in the SIW case. However, the comparison is not so straightforward since SIW employs RT/duroid 5880 with lower loss tangent than RO3003 and RO4003 at $60 \mathrm{GHz}$. Thereby, we could expect even smaller losses in inverted microstrip gap waveguide than SIW if designed with same dielectric material.

\section{ACKNOWLEDGMENT}

This work was supported by the following four parts: the Swedish Research Council VR, the Swedish Governmental Agency for Innovation Systems VINNOVA via a project within the VINN Excellence center Chase, the European Research Council (ERC) via the advanced investigator grant ERC-2012-ADG20120216, and the Spanish Government under project TEC2013-44019-R. The authors would like to thank Prof. Vessen Vassilev from the Microtechnology and Nanoscience department at Chalmers University of Technology (Sweden) for his useful advices to this project, as well as help during the setup of the measurement equipment and assembling of the gap waveguide prototypes.

\section{REFERENCES}

[1] F. Gardiol, "Radiation from microstrip circuits: An Introduction", International Journal of Microwave and Millimeter-Wave Computer-Aided Engineering, vol. 1, no. 2, pp. 225-235, 1991

[2] E. Levine, G. Malamud, S. Shtrikman, and D. Treves, "A study of microstrip array antennas with the feed network", IEEE Transactions on Antennas and Propagation, vol. 37, no. 4, pp. 426-434, 1989.

[3] Y. Miura, J. Hirokawa, M. Ando, Y. Shibuya, and G. Yoshida, "Double layer full-corporate-feed hollow-waveguide slot array antenna in the 60 GHz-band", IEEE Transactions on Antennas and Propagation, vol. 59, no. 8 , pp. 2844-2851, August 2011. 
[4] S.S. Oh, J. W. Lee, M. S. Song, and Y. S. Kim, "Two-layer slotted waveguide antenna array with broad reflection/gain bandwidth at millimeterwave frequencies", IEE Proc.-Microw. Antennas Propag., vol. 51, no. 5, pp. 393-398, October 2004.

[5] D. Deslandes and K. Wu, "Accurate modeling, wave mechanisms, and design considerations of a substrate integrated waveguide", IEEE Transactions on Microwave Theory and Techniques, vol. 54, no. 6, pp. 25162526, June 2006.

[6] $\mathrm{F} . \mathrm{Xu}$ and $\mathrm{K}$. Wu, "Guided-wave and leakage characteristics of substrate integrated waveguide", IEEE Transactions on Microwave Theory and Techniques, vol. 53, no. 1, pp. 66-73, January 2005.

[7] M. Bozzi, F. Xu, D. Deslandes and K. Wu, "Modelling and design considerations for substrate integrated waveguide circuits and components", in Proceedings of the $8^{\text {th }}$ International Conference on Telecommunications in Modern Satellite, Cable and Broadcasting Services. TELSIKS 2007.

[8] L. Yan, W. Hong, G. Hua, J. Chen, K. Wu, and T.J. Cui, "Simulation and experiment on SIW slot array antennas", IEEE Microwave and Wireless Components Letters, vol. 14, no. 9, pp. 446-448, September 2004.

[9] D.-F. Guan, Z.-P. Qian, Y.-S. Zhang, and Y. Cai, "Novel SIW cavitybacked antenna array without using individual feeding network", IEEE Antennas and Wireless Propagation Letters, vol. 13, pp. 423-426, 2014.

[10] P.-S. Kildal, "Artificially soft and hard surfaces in electromagnetics", IEEE Transaction on Antennas and Propagation, vol. 28, no. 10, pp. 1537-1544, 1990

[11] P.-S. Kildal, E. Alfonso, A. Valero-Nogueira, and E. Rajo-Iglesias, "Local metamaterial-based waveguides in gaps between parallel metal plates", IEEE Antennas and Wireless Propagation Letters, vol. 8, pp. 8487, 2009.

[12] P.-S. Kildal, "Three metamaterial-based gap waveguides between parallel metal plates for $\mathrm{mm} / \mathrm{submm}$ waves", in Proceedings of the $3^{\text {rd }}$ European Conference on Antennas and Propagation EUCAP, Berlin, Germany, 23-27 March 2009.

[13] A. Valero-Nogueira, E. Alfonso, J. I. Herranz, and P.-S. Kildal, "Experimental demonstration of local quasi-TEM gap modes in single-hard-wall waveguides", IEEE Microwave and Wireless Components Letters, vol. 19, no. 9, pp. 536-538, September 2009.

[14] P.-S. Kildal, A. U. Zaman, E. Rajo-Iglesias, E. Alfonso, and A. ValeroNogueira, "Design and experimental verification of ridge gap waveguides in bed of nails for parallel plate mode suppression", IET Microwaves, Antennas \& Propagation, vol. 5, no 3, pp. 262-270, March 2011.

[15] E. Pucci, A. U. Zaman, E. Rajo-Iglesias, P.-S. Kildal and A. Kishk, "Study of Q-Factors of Ridge and Groove Gap Waveguide Resonators", IET Microwaves, Antennas \& Propagation, vol. 7, no. 11, pp. 900-908, August 2013.

[16] A. U. Zaman, T. Vukusic, M. Alexanderson, and P.-S. Kildal, "Design of a simple transition from microstrip to ridge gap waveguide suited for MMIC and antenna integration", IEEE Antennas and Wireless Propagation Letters, vol. 12, pp. 1558-1561, 2013.

[17] A. Algaba Brazález, A. U. Zaman, and P.-S.Kildal, "Design of a coplanar waveguide-to-ridge gap waveguide transition via capacitive coupling", in Proceedings of the $6^{\text {th }}$ European Conference on Antennas and Propagation EUCAP, Prague, Czech Republic, 26-30 March 2012.

[18] A. Algaba Brazález, A. U. Zaman, and P.-S. Kildal, "Investigation of a Microstrip-to-Ridge Gap Waveguide transition by electromagnetic coupling", in Proceedings of the IEEE Antennas and Propagation Society International Symposium, pp. 1-2, 2012.

[19] E. Pucci, A. U. Zaman, E. Rajo-Iglesias, and P.-S. Kildal, "New low loss inverted microstrip line using gap waveguide technology for slot antenna applications", in Proceedings of the $5^{\text {th }}$ European Conference on Antennas and Propagation EUCAP, Rome, Italy, 11-15 April 2011.

[20] A. Valero-Nogueira, M. Baquero, J. I. Herranz, J. Domenech, E. Alfonso and A. Vila, "Gap waveguides using a suspended strip on a bed of nails", IEEE Antennas and Wireless Propagation Letters, vol. 10, pp. 1006-1009, 2011

[21] E. Rajo-Iglesias and P.-S. Kildal, "Numerical studies of bandwidth of parallel plate cut-off realized by bed of nails, corrugations and mushroomtype EBG for use in gap waveguides", IET Microwaves, Antennas \& Propagation, vol. 5, no 3, pp. 282-289, March 2011.

[22] E. Pucci, E. Rajo-Iglesias, J.-L. Vázquez-Roy and P.-S. Kildal, "Planar Dual-Mode Horn Array with Corporate-Feed Network in Inverted Microstrip Gap Waveguide", IEEE Transactions on Antennas and Propagation, vol. 62, issue 7, pp. 3534-3542, 2014.

[23] C. Gahete Arias, M. Baquero Escudero, A. Valero Nogueira, and A. Vila Jiménez, "Test-Fixture for Suspended-Strip Gap-Waveguide Technology on Ka-Band", IEEE Microwave and Wireless Components Letters, vol. 23, pp. 321-323, 2013.
[24] H. Raza, J. Yang, P.-S. Kildal, and E. Alfonso, "Microstrip-ridge gap waveguide - study of losses, bends and transition to WR-15", IEEE Transactions on Microwave Theory and Techniques, vol. 62, issue. 9, pp. 1943-1952, 2014.

[25] S. A. Razavi, P.-S. Kildal, L. Xiang, E. Alfonso, and H. Chen, "2x2-slot element for $60 \mathrm{GHz}$ planar array antenna realized on two doubled-sided PCBs using SIW cavity and EBG-type soft surface fed by microstripridge gap waveguide", IEEE Transactions on Antennas and Propagation, vol. 62, issue. 9, pp. 4564-4573, 2014.

[26] A. Algaba Brazález, E. Rajo-Iglesias, and P.-S. Kildal, "Investigation of transitions for use in inverted microstrip gap waveguide antenna arrays", in Proceedings of the $8^{\text {th }}$ European Conference on Antennas and Propagation EUCAP, The Hague, Netherlands, 6-11, April 2014.

[27] B. Boukari, E. Moldovan, S. Affes, K. Wu, R. G. Bosisio, and S. O. Tatu, "Robust microstrip-to-waveguide transitions for millimeter-wave radar sensor applications", IEEE Antennas and Wireless Propagation Letters, vol. 8, pp. 759-762, 2009.

[28] K. Y. Han and C.-K. Pao, "A V-band waveguide to microstrip inline transition", IEEE MTT-S Dig., pp. 1-3, 2012.

[29] P. Hallbjörner, "Circuit board integrated patch transition between microstrip and rectangular waveguide, optimised for producibility", IEE Proceedings Microwaves, Antennas and Propagation, vol. 153, issue 4, pp. 330-334, 2006.

[30] Y.-C. Shih, T.-N- Ton, and L. Q. Bui, "Waveguide-to microstrip transitions for millimeter-wave applications", IEEE MTT-S Dig., pp. 473-475, 1998.

[31] Y. Tikhov, J.-W. Moon, Y.-J. Kim, Y. Sinelnikov, "Refined characterization of E-plane waveguide to microstrip transition for millimeter-wave applications", in Proceedings of the Asia-Pacific Microwave Conference, 2000.

[32] Y.-C. Leong and S. Weinreb, "Full band waveguide-to-microstrip probe transitions", IEEE MTT-S International Microwave Symposium Digest, 1999.

[33] A. Artemenko, A. Maltsev, R. Maslennikov, A. Sevastyanov, and V. Ssoring, "Design of wideband waveguide to microstrip transition for 60 $\mathrm{GHz}$ frequency band", the $41^{\text {st }}$ European Microwave Conference (EuMC), pp. 838-841, 2011.

[34] K. Seo, K. Sakakibara, and N. Kikuma, "Microstrip-to-waveguide transition using waveguide with large broad-wall in millimeter-wave band", in Proceedings of the IEEE International Conference on Ultra-Wideband (ICUWB), pp. 1-4, 2010

[35] A. L. Vera-López, S.K. Bhattacharya, C.A. Donado-Morcillo, J. Papapolymerou, D. Choudhury, and A. Horn, "Novel los loss thin film materials for wireless $60 \mathrm{GHz}$ application", in Proceedings of the $60^{\text {th }}$ IEEE Electronic Components and Technology Conference (ECTC), 2010.

[36] L. Yujian and L. Kwai-Man, "A broadband V-band rectangular waveguide to substrate integrated waveguide transition", IEEE Microwave and Wireless Components Letters, vol. 24, no. 9, pp. 590-592, September 2014.

[37] A. Bakhtafrooz and A.Borji, "Novel two-layer millimeter-wave slot array antennas based on substrate integrated waveguide", Progress In Electromagnetics Research, vol.109, pp. 475-491, 2010.

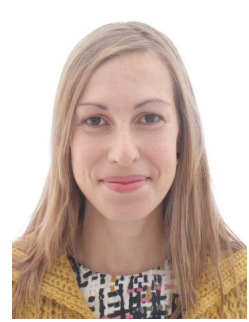

Astrid Algaba Brazález was born in Alicante, Spain, in 1983. She received her Telecommunication Engineering degree from Miguel Hernández University of Elche, Alicante, Spain, in 2009, and the Licentiate of Engineering and $\mathrm{PhD}$ degrees from Chalmers University of Technology, Gothenburg, Sweden, in 2013 and 2015, respectively. She has joined Ericsson Research, Ericsson AB, Gothenburg, Sweden, in November 2014

Her main research interests include the development of gap waveguide technology for millimeter and sub-millimeter wave applications, microwave passive gap waveguide components, packaging of microstrip filters, design of high frequency transitions between gap waveguide and other technologies, and metamaterials. 


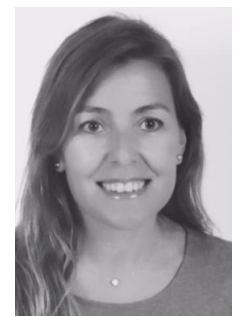

Eva Rajo-Iglesias was born in Monforte de Lemos, Spain, in 1972. She received the M.Sc. degree in telecommunication engineering from the University of Vigo, Spain, in 1996, and the Ph.D. degree in telecommunication engineering from the University Carlos III of Madrid, Spain, in 2002.

She was a Teacher Assistant with the University Carlos III of Madrid from 1997 to 2001. She joined the Polytechnic University of Cartagena, Cartagena, Spain, as a Teacher Assistant, in 2001. She joined University Carlos III of Madrid as a Visiting Lecturer in 2002, where she has been an Associate Professor with the Department of Signal Theory and Communications since 2004. She visited the Chalmers University of Technology, Göteborg, Sweden, as a Guest Researcher, in 2004 , 2005, 2006, 2007, and 2008, and has been an Affiliate Professor with the Antenna Group, Signals and Systems Department, since 2009. She has coauthored more than 50 papers in JCR international journals and more than 100 papers in international conferences. Her current research interests include microstrip patch antennas and arrays, metamaterials, artificial surfaces and periodic structures, MIMO systems and optimization methods applied to electromagnetism

Dr. Rajo-Iglesias was the recipient of the Loughborough Antennas and Propagation Conference Best Paper Award in 2007, the Best Poster Award in the field of Metamaterial Applications in Antennas, at the conference Metamaterials 2009, the 2014 Excellence Award to Young Research Staff at the University Carlos III of Madrid and the Third Place Winner of the Bell Labs Prize 2014. She is currently an Associate Editor of the IEEE ANTENNAS AND PROPAGATION MAGAZINE and of the IEEE ANTENNAS AND WIRELESS PROPAGATION LETTERS.

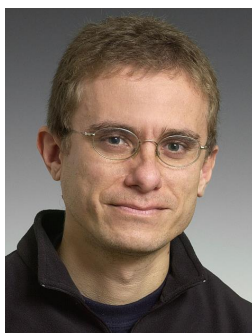

José Luis Vázquez-Roy was born in Madrid, Spain, in 1969. He received the Ingeniero de Telecomunicacion and Ph.D. degrees from the Universidad Politecnica de Madrid, Madrid, Spain, in 1993 and 1999, respectively. In 1999, he joined the "Teoria de la Se nal y Comunicaciones" Department, Universidad Carlos III de Madrid, Spain, where he is currently an Associate Professor.

His research activities and interests include the analysis and design of planar antennas, artificial surfaces, and periodic structures for microwave and millimeter wave applications.

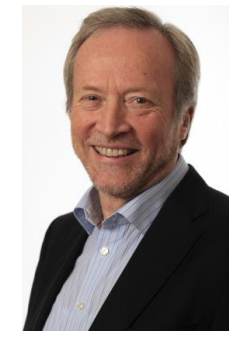

Per-Simon Kildal has been Professor at Chalmers University of Technology, Gothenburg, Sweden since 1989. He is now heading the Division of Antenna Systems at Department of Signals and Systems at Chalmers.

Prof Kildal received two best paper awards for articles published in the IEEE Transactions on Antennas and Propagation, and he was the recipient of the 2011 Distinguished Achievements Award of the IEEE Antennas and Propagation Society. Kildal has authored an antenna textbook, and more than 150 journal articles and letters, most of them in IEEE or IET journals. He has designed two very large antennas, including the Gregorian dual-reflector feed of the Arecibo radiotelescope. He has invented several reflector antenna feeds, the latest being the so-called "Eleven antenna".

Kildal is the originator of the concept of soft and hard surfaces, recently resulting in the gap waveguide, a new low-loss metamaterial-based transmission line advantageous in particular above $30 \mathrm{GHz}$. Kildal has received large individual grants from the Swedish research council VR and from the European Research Council ERC for research on gap waveguides.

His research group has pioneered the reverberation chamber into an accurate measurement tool for antennas and wireless terminals subject to Rayleigh fading. This has been successfully commercialized in Bluetest AB.

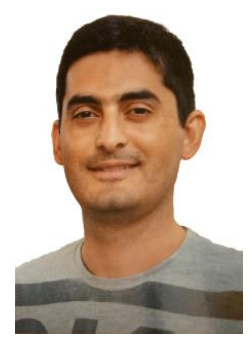

Abbas Vosoogh received the B.Sc. degree in electrical engineering from the University of Sistan and Baluchestan, Zahedan, and the M.Sc. degree from the K. N. Toosi University of Technology, Tehran, Iran. He is currently pursuing the Ph.D. degree with Chalmers University of Technology, Gothenburg, Sweden.

His current research interests include millimeter and submillimeter wave guiding structures, EBG, soft and hard surfaces and planar array antennas. 